Research Paper

\title{
Keratin 80 regulated by miR-206/ETS1 promotes tumor progression via the MEK/ERK pathway in ovarian cancer
}

\author{
Ouxuan Liu'1,2, Caixia Wang33, Shuang Wang1,2, Yuexin $\mathrm{Hu}^{1,2}$, Rui Gou ${ }^{1,2}$, Hui Dong1,2, Siting Li1,2, Xiao Li1,2, \\ Bei Lin ${ }^{1,2}$ \\ 1. Department of Obstetrics and Gynecology, Shengiing Hospital of China Medical University, Liaoning, China. \\ 2. Key Laboratory of Maternal-Fetal Medicine of Liaoning Province, Key Laboratory of Obstetrics and Gynecology of Higher Education of Liaoning Province, \\ Liaoning, China. \\ 3. Department of Obstetrics and Gynecology, West China Second University Hospital, Sichuan University, Chengdu, China. \\ $\triangle$ Corresponding author: Bei Lin, Department of Obstetrics and Gynecology, Shengjing Hospital Affiliated to China Medical University, No. 36, Sanhao Street, \\ Heping District, Liaoning, Shenyang 110004, People's Republic of China. E-mail: linbei88@hotmail.com.
}

() The author(s). This is an open access article distributed under the terms of the Creative Commons Attribution License (https://creativecommons.org/licenses/by/4.0/). See http://ivyspring.com/terms for full terms and conditions.

Received: 2021.08.18; Accepted: 2021.09.18; Published: 2021.09.24

\begin{abstract}
Introduction: Keratin 80 (KRT80) is a type II epithelial keratin protein that plays an important role in cell differentiation and tumor progression. However, its role and mechanisms in ovarian cancer remain unclear.

Methods: The effect of KRT80 on the survival and prognosis of patients with ovarian cancer was determined using immunohistochemistry. Cell lines overexpressing KRT80 and with KRT80 knockdown were established to study its effect on the malignant behavior of ovarian cancer cells. Western blotting was used to detect changes in related molecules, and in the MEK/ERK signal transduction pathway. ChIP assay was used to confirm that ETSI regulates KRT80 at the transcriptional level. A double luciferase assay was used to confirm the target of miR-206.

Results: The expression levels of KRT80 were high in ovarian cancer tissue, and were related to survival and prognosis. KRT80 expression is an independent prognostic factor in patients with ovarian cancer. KRT80 overexpression promotes the proliferation of ovarian cancer cells, the transition from G1 phase to $S$ phase, invasion, and migration. KRT80 overexpression increased the expression of BCL2/BAX, CyclinD1, MMP2, MMP9, and N-cadherin, decreased the expression of E-cadherin, and increased the phosphorylation of MEK and ERK. ETSI binds to the upstream promoter sequence of KRT80 and regulates KRT80 expression at the transcriptional level. ETS1 is a direct target of miR-206 in ovarian cancer cells.

Conclusion: KRT80 regulated by miR-206/ETS1 promotes tumor progression via the MEK/ERK pathway in ovarian cancer, and KRT80 may have applications as a screening biomarker and potential therapeutic target for ovarian cancer.
\end{abstract}

Key words: ovarian cancer; KRT80; MEK/ERK pathway; ETS1; miR-206

\section{Introduction}

Ovarian cancer $(\mathrm{OC})$ is one of the most common gynecologic malignancies worldwide, and is the leading cause of death due to gynecologic malignancies $[1,2]$. The 5 year survival rate for ovarian cancer patients is approximately $47 \%$, whereas the 5 year survival rate for stage III and IV ovarian cancer patients is only $29 \%[3,4]$. Due to a lack of effective and sensitive clinical screening methods for early ovarian cancer, it is urgent to further explore specific tumor markers to predict the occurrence and outcome of ovarian cancer. Therefore, it is vital to search for genes involved in the occurrence, development, and chemotherapy resistance of ovarian cancer, and to explore their applicability in the early diagnosis, disease monitoring, and prognosis evaluation of ovarian cancer. 
The KRT80 gene is located at the centromeric end of the human type II keratin gene domain on chromosome 12q13.13, and encodes a 452-amino acid protein with a molecular weight of $50.5 \mathrm{kDa}[5,6]$. KRT80 is involved in cell differentiation, and is located near the desmosomal plaques during the early stage of differentiation, and dispersed throughout the cytoplasm in terminal differentiated cells. Keratin is an intermediate filament cytoskeletal protein that maintains the structural integrity of epithelial cells [7], and can be classified into epithelial and hair keratin. The expression profile of KRT80 suggests that it encodes a type II epithelial keratin, whereas the protein it encodes is more structurally similar to type II hair keratin [8]. Keratin has tissue specificity, and is expressed in a differentiation-dependent manner [9]. Keratin is widely used as a tumor marker in cancer diagnosis, and plays an active biological role in tumor cell proliferation and metastasis [10]. Recently, abnormal KRT80 expression has been found in colorectal, gastric, and breast cancer, where it plays an important role in tumor development and progression [11-14]. However, KRT80 expression in ovarian cancer, and its effect on malignant biological behavior, have not been reported.

ETS1 is a nuclear protein that mainly acts as a transcription activator, and is involved in the development of stem cells, cell senescence and apoptosis, and tumorigenesis [15]. ETS1 contributes to tumor angiogenesis and invasion and migration of cancer cells, and promotes epithelial-mesenchymal transition (EMT) and the development of drug resistance. It is closely related to the occurrence, development, metastasis, and prognosis of tumors, and is overexpressed in many types of tumors $[16,17]$. The microRNA (miRNA) miR-206 is located on human chromosome $6 \mathrm{p} 12.2$, and impacts posttranscriptional regulation of gene expression in multicellular organisms by affecting mRNA stability and translation [18]. Because it is downregulated in a variety of tumors, miR-206 is assumed to be a tumor suppressor [19], and also affects tumor cell proliferation, differentiation, invasion, metastasis, and other processes by regulating genes related to cell cycle, division, and apoptosis. For these reasons, miR-206 expression could be a biomarker of disease status and prognosis, and a predictor of drug resistance $[20,21]$.

In the present study, we examined the expression of KRT80 in ovarian tissue, and studied the relationship between KRT80 expression and clinicopathologic parameters and prognosis of ovarian cancer. We also studied the effect of KRT80 overexpression and knockdown on the malignant biological behavior of ovarian cancer cells in vitro. We further explored the relationship between the expression of KRT80 and the occurrence and development of ovarian cancer, revealing its regulatory pathways, transcription factors, and upstream miRNAs. These findings provide a new theoretical basis for further study of the mechanisms by which KRT80 participates in the occurrence and development of ovarian cancer. KRT80 may have applications as a diagnostic and prognostic indicator for ovarian cancer, and may represent a therapeutic target for novel targeted treatment regimens.

\section{Methods}

\section{Source of specimen and clinical data}

A total of 147 paraffin-embedded pathological specimens were collected from 2008 to 2014 at the Department of Obstetrics and Gynecology, Shengjing Hospital, China Medical University. According to the ethical and legal standards, all selected patients have obtained written informed consent. The study was approved by the Ethics Review Committee of Shengjing Hospital, China Medical University. All the ovarian malignant tumor samples were primary epithelial ovarian tumors. Normal ovarian tissues were from postmenopausal patients or from cervical cancer patients with double adnexal hysterectomy. All the cases have complete clinical data which can be obtained for each patient. Samples from patients who had received radiation therapy, chemotherapeutic, and hormone one therapy were excluded from the cohort. The pathological diagnosis of all tissue sections was performed by a Sheng Jing Hospital pathologist, and this sample set included 102 cases of ovarian epithelial malignant tumors (ovarian cancer group), 16 cases of ovarian epithelial borderline tumors (ovarian borderline group), 14 cases of benign tumors (ovarian benign group), and 15 cases of normal ovarian tissue (ovarian normal group). The mean ages of patients in each group were: 53.2 years in the ovarian cancer group (range: 16-79 years), 49.5 years in the borderline group (range: 19-84 years), 48.14 years in the benign group (range: $16-78$ years), and 47.8 years in the normal group (range: $32-76$ years). There was no significant difference in age between the groups $(P>0.05)$. In the ovarian cancer group, there were 67 cases of serous cystadenocarcinoma, 8 cases of mucinous adenocarcinoma, 19 cases of endometrioid adenocarcinoma, and 8 cases of clear cell carcinoma. According to histological classification, 27 cases were highly differentiated, 25 cases were moderately differentiated, and 50 cases had low differentiation. According to the International Federation of Obstetrics and Gynecology (FIGO, 2009), there were 
24 cases in stage I, 19 cases in Stage II, 54 cases in stage III, and five cases in stage IV. There were 22 and 65 cases with and without lymph node metastasis, respectively, and 15 cases without lymph node dissection.

\section{Immunohistochemistry}

Paraffin-embedded tissue samples from each group were fixed in $10 \%$ formalin and sectioned continuously at a thickness of $5 \mu \mathrm{m}$ after conventional paraffin embedding. KRT80 expression was detected using an ultra-sensitive TM SP (mouse/rabbit) IHC kit (Maixin, China, Cat\# KIT-9720). Rabbit KRT80 polyclonal antibody (Proteintech, Wuhan, China, Cat\# 16835-1-AP) was used at a 1:500 dilution. Brown and yellow granules were observed in the cytoplasm, and were considered to be positive staining. A staining intensity score was adopted, with 0 indicating no coloring, 1 indicating light yellow, 2 indicating brown yellow, and 3 indicating brown. The percentage of stained cells in a visual field was scored according to the following: 0 (<5\%), 1 (5 25\%), 2 (26 $\sim 50 \%), 3(51 \sim 75 \%)$, and $4(>75 \%)$. The final score was calculated as the product of the staining score and percentage of stained cells in a visual field, and was denoted as follows: $0 \sim 2(-), 3 \sim 4(+), 5 \sim 8(++)$, and $9 \sim 12(+++)$. Based on these scores, $0 \sim 4$ was designated as the 'low expression group' and $5 \sim 12$ as the 'high expression group'. Each sample was scored by two independent observers, and disagreements were resolved by a third independent observer.

\section{Cell culture}

Ovarian cancer cell lines (CAOV3 and OVCAR3) and normal ovary epithelial cells (HOSEpiC) were purchased from the Cell Bank of the Chinese Academy of Sciences (Shanghai Institute of Biochemistry and Cell Biology, Shanghai, China). CAOV3, OVCAR3 and HOSEpiC cells were cultured in normal RPMI1640 medium (BI, USA) containing $10 \%$ fetal bovine serum (FBS). The cells were cultured at $37^{\circ} \mathrm{C}$ under $5 \% \mathrm{CO}_{2}$ and saturated humidity.

\section{Cell transfection and construction of stable transfected cell lines}

Cells in the logarithmic growth phase were inoculated into 6-well plates the day before transfection. KRT80 small interfering RNA (siRNA) was constructed by GenePharma (Shanghai, China) to knock down KRT80. The KRT80 siRNA and negative control siRNA were transfected into CAOV3 and OVCAR3 cells, using lipofectamine (Lipofectamine 3000 transfection kit, GIBCO, Invitrogen). The sequence of KRT80 siRNA is: sense: 5'-GCUCCUGC GUGGUUGGCUUTT-3', antisense: 5'-AAGCCAACC ACGCAGGAGCTT-3'. The sequence of its negative control is: sense: 5'-UUCUCCGAACGUGUCACGU TT-3', antisense: 5'-ACGUGACACGUUCGGAGAA TT-3'. After transfection for $48 \mathrm{~h}$, the cells were collected for analysis via real-time quantitative PCR (RT-qPCR), western blotting, and biological assays. The KRT80 overexpressing cell line and associated negative control cell line were constructed by transfecting CAOV3 and OVCAR3 cells using the GeneChem lentivirus gene transfection system. Stable cell lines were screened with $2 \mu \mathrm{g} / \mathrm{ml}$ puromycin (Solarbio, Beijing, China).

\section{RT-qPCR}

Total RNA was extracted from transfected cells using the TRIzol reagent (Takara Bio, Inc., Shiga, Japan) according to the manufacturer's instructions. The purity and concentration of RNA were determined by ultraviolet spectrophotometer. RNA was reverse transcribed into cDNA using the Takara 047A kit (Takara Bio, Inc., Shiga, Japan), and RT-qPCR was performed using a 7500 Fast real-time PCR system. The amplification conditions were: denaturation at $95^{\circ} \mathrm{C}$ for $30 \mathrm{~s}, 95^{\circ} \mathrm{C}$ for $5 \mathrm{~s}$, and $60^{\circ} \mathrm{C}$ for $30 \mathrm{~s}$, for a total of 40 cycles. KRT80 primer sequence: Forward: 5'-AACCAGGAGAAGGAGGAG ATGAAGG-3', Reverse: 5'-CCAGTTCAGTCCGATG AAGACACTC-3'. GAPDH primer sequence: Forward: 5'-ACAACTTTGGTATCGTGGAAGG-3', Reverse: 5'-GCCATCACGCCACAGTTTC-3'. The abundance of KRT 80 mRNA was determined using the comparative $-\Delta \mathrm{Ct}$ method.

\section{Western blotting}

Cells were lysed via ultrasonication in RIPA cell lysis buffer at $4{ }^{\circ} \mathrm{C}$ for $30 \mathrm{~min}$. The cell debris was pelleted via centrifugation at $4{ }^{\circ} \mathrm{C}$ for $30 \mathrm{~min}$ at 12000 $\mathrm{rpm}$, and the supernatant containing the total protein was collected into a new Eppendorf tube. The total protein concentration was determined using a BCA assay. Loading buffer was added to each sample and the protein was denatured at $100{ }^{\circ} \mathrm{C}$ for $5 \mathrm{~min}$. The protein samples were separated electrophoretically using $10 \%$ sodium dodecyl sulphate-polyacrylamide gel electrophoresis, and then transferred onto a polyvinylidene fluoride membrane for western blotting. The membrane was subsequently blocked for $2 \mathrm{~h}$ using $5 \%$ milk or bovine serum albumin, and incubated overnight at $4{ }^{\circ} \mathrm{C}$ with primary antibodies in blocking solution. The primary antibodies we used are as follows: KRT80 (Proteintech, rabbit polyclonal antibody, 1:1000), BCL2 (Proteintech, rabbit polyclonal antibody, 1:2000), BAX (Affinity, rabbit polyclonal antibody, 1:1000), CyclinD1 (CST, rabbit polyclonal antibody, 1:1000), MMP2 (Proteintech, rabbit polyclonal antibody, 1:1000), MMP9 (Protein- 
tech, rabbit polyclonal antibody, 1:500), E-Cadherin (Proteintech, rabbit polyclonal antibody, 1: 2000), N-Cadherin (Proteintech, rabbit polyclonal antibody, 1:2000), MEK (Affinity, rabbit polyclonal antibody, 1:1000), p-MEK (Affinity, rabbit polyclonal antibody, 1:1000), ERK (CST, rabbit polyclonal antibody, 1:1000), p-ERK (CST, rabbit polyclonal antibody, 1:1000), ETS1 (CST, rabbit polyclonal antibody, 1:1000), GAPDH (ZSGB-BIO, mouse monoclonal antibody, 1:2000). The next day, the membrane was washed 3 times with $1 \times$ TBST for $10 \mathrm{~min}$ each. HRPlabeled goat anti-rabbit secondary antibody or murine IgG (1:2000, Zhongshan Jinqiao, China) was added to the membranes and incubated for $2 \mathrm{~h}$, and then washed with $1 \times$ TBST 3 times for $10 \mathrm{~min}$ each. Protein expression was determined using a chemiluminescent HRP substrate (Millipore, Billerica, MA, USA).

\section{Cell proliferation test}

Adhered cells were resuspended by tryptic digestion, and then diluted to $2 \times 10^{4}$ cells $/ \mathrm{ml}$ and inoculated into 96-well plates at 2000 cells/well. The initial time point, $0 \mathrm{~h}$, was defined as the time when the suspended cells adhered to the wells. To measure cell proliferation, $10 \mu \mathrm{l}$ of CCK8 reagent (Vazyme Biotech, Nanjing, China) was added to each well and incubated for $0,24,48,72$, and $96 \mathrm{~h}$ at $37^{\circ} \mathrm{C}$ for $4 \mathrm{~h}$. The optical density at $450 \mathrm{~nm}$ was measured at each time point.

\section{Cell cycle}

Cells were removed from plates by digesting with trypsin without ethylenediaminetetraacetic acid (EDTA), and cell suspensions were collected by centrifugation at $1000 \mathrm{rpm}$ for $5 \mathrm{~min}$. The supernatant was removed, and $500 \mu \mathrm{l}$ of pre-cooled $70 \%$ ethanol was added to fix the cells overnight at $4{ }^{\circ} \mathrm{C}$. The next day the ethanol was removed by centrifugation, and the cells were washed with PBS. Thereafter, $500 \mu l$ of 9:1 PI/RNaseA staining solution (KeyGen Bio-tech, Nanjing, China) was added to each sample. The color was developed by incubating cells at room temperature for $30 \mathrm{~min}$. The proportion of cells in the G0/G1, S, or G2/M phase was measured using flow cytometry on a BD FACSDiva system (BD Biosciences, New York, USA).

\section{Scratch test}

Cell suspensions were prepared from logarithmic growth phase cells and inoculated into 6-well plates. When the cells grew to $90 \%$ confluency, the tip of a $200 \mu$ pipette tip was used to gently scratch the surface of the 6-well plates. After washing 2 3 times with PBS, the cell fragments were removed and cultured in serum-free medium. The scratch width was observed and measured at $0 \mathrm{~h}$ and $24 \mathrm{~h}$ under a microscope.

\section{Transwell assay}

Matrigel (BD Biosciences, USA) and serum-free medium were mixed at a ratio of 1:7.5, and $70 \mu \mathrm{l}$ was added to the upper chambers of each transwell chamber (Corning Coster) and placed in an incubator at $37{ }^{\circ} \mathrm{C}$ overnight. Thereafter, $500 \mu \mathrm{l}$ of RPMI1640 medium containing 20\% FBS was added to the lower chamber, and $200 \mu \mathrm{l}$ of serum-free cell suspension $\left(2 \times 10^{5}\right.$ cells $\left./ \mathrm{ml}\right)$ was added to the upper chamber. The cells were incubated at $37^{\circ} \mathrm{C}$ under $5 \% \mathrm{CO}_{2}$ for 24 or $48 \mathrm{~h}$. Thereafter, cells were washed with PBS and fixed in $4 \%$ paraformaldehyde for $30 \mathrm{~min}$, washed again with PBS, and stained with $0.1 \%$ crystal violet for $30 \mathrm{~min}$. The upper chamber surface was lightly swabbed with a cotton swab, and the number of ovarian cancer cells infiltrating the chamber was counted under a microscope.

\section{Chromatin immunoprecipitation (ChIP)}

Ovarian cancer cells in the logarithmic phase were collected. The ChIP assay was performed using the Simple ChIP Enzymatic Chromatin IP Kit (\#9004, Cell Signaling Technology, California, USA) according to the manufacturer's instructions. Cell samples were processed by preparation of the nuclei, digestion and immunoprecipitation the chromatin, immune complex precipitation and washing, elution of the chromatin, and reversal of cross-linking and DNA purification. ETS1 was visualized by the addition of $10 \mu \mathrm{l}$ of ETS1 antibody (CST, [rabbit], 14069S) and $2 \mu \mathrm{g}$ of IgG antibody (CST, [mouse], 5415S). DNA from the immunoprecipitation was subsequently amplified via RT-qPCR using the specific primer. The binding site primer sequence: Forward: 5'-TGACCGTCTATGTCCTCC-3', Reverse: 5'-CTCCCTGGCTTATCTTCC-3'.

\section{Dual luciferase reporter gene assay}

The plasmid for the double luciferase reporter gene assay was synthesized by GenePharma (Shanghai, China). A wild-type (WT) fragment (ETS1-WT), and corresponding mutant (Mut) fragment (ETS1-MUT), containing the miR-206 potential binding site were inserted into the reporter vector to construct the ETS1 luciferase reporter vector. HEK293T cells were cultured on a 24-well plate and co-transfected with miR-206 mimic or miR-Mock and WT or Mut report vector when $70 \%$ of HEK293T cells were fused. After transfection for 48 hours, whole cell lysate was collected and luciferase activity was measured by luciferase reporter assay (Promega, Madison, WI, USA), according to the manufacturer's instructions. 


\section{Bioinformatics}

The Oncomine database (http://www. oncomine.org) was used to analyze the expression of KRT80 mRNA in different types of cancer. GEPIA (http://gepia.cancer-pku.cn/) was used to analyze the differential expression of KRT80 in ovarian cancer and normal ovarian tissue. The changes in KRT80 expression in patients with ovarian cancer were assessed using the cBioPortal repository (http://www.cbioportal.org), and the co-expressed genes were screened. The prognostic utility of KRT80 in ovarian cancer was studied using Kaplan-Meier survival plots (http://kmplot.com). Functional and pathway enrichment analysis of KRT80-related genes was performed using the David database (https://david.ncifcrf.gov), which provides a systematic and comprehensive biological functional annotation. The C2.cp.kegg.v6.2.symbols.gmt dataset was downloaded from the MsigDB database on the gene set enrichment analysis (GSEA) website, and the GSEA 3.0 software was used for genome enrichment analysis. The Promo database (http://alggen.lsi. upc.es/cgibin/promo_v3/promo/promoinit.cgi?dir $\mathrm{DB}=\mathrm{TF} \_8.3$ ) and JASPAR database (http://jaspar. genereg.net/) were then used to predict the transcription factor/s of KRT80, and the StarBase database (http://starbase.sysu.edu.cn/) was used to analyze the correlation between ETS1 mRNA and KRT80 mRNA. The TargetScan database (http:// www.targetscan.org/) and miRDB database (http:// mirdb.org/) were used to predict miRNAs upstream of ETS1, and possible binding sites.

\section{Statistical analysis}

The data were analyzed using SPSS 21.0 (IBM Corporation, Armonk, NY, USA), and graphs was generated in GraphPad Prism 8.0. All data are represented as the mean \pm SD. T-tests and chi-squared tests were used to compare the differences between two groups. One-way analysis of variance (ANOVA) was used for comparison between two or more groups, and Kaplan-Meier and log-rank tests were used for survival curve analysis. Univariate and multivariate Cox regression models were used to analyze the risk factors that affect prognosis. A bilateral $P<0.05$ was considered to indicate statistical significance. ${ }^{*} *, P<0.001 ; * *, P<0.01 ;{ }^{*}, P<0.05$.

\section{Results}

\section{Expression and clinical significance of KRT80 in different ovarian tissues}

KRT80 protein was primarily expressed in the cytoplasm (Figure 1A), and was expressed in ovarian tissue in all groups. KRT80 expression increased gradually with the progression of malignant ovarian cancer. The positive expression rate of KRT80 in the ovarian cancer group $(79.41 \%, 81 / 102)$ was significantly higher than that in the normal $(26.67 \%$, $4 / 15)$ and benign $(28.57 \%, 4 / 14)$ groups $(P<0.001)$. The positive expression rate of KRT80 in the ovarian cancer group $(79.41 \%, 81 / 102)$ was also higher than that in ovarian borderline group $(75.00 \%, 12 / 16)$, but the difference was not statistically significant $(P>$ 0.05). We used a scoring metric to define the expression of KRT80, with $(++)$ and $(+++)$ denoting high expression. The high expression rate of KRT80 in the ovarian cancer group $(56.86 \%, 58 / 102)$ was significantly higher than that in the normal $(0)$ and benign $(7.14 \%, 1 / 14)$ groups $(P<0.001)$. The high expression rate of KRT80 in the ovarian cancer group $(56.86 \%, 58 / 102)$ was also higher than that in the ovarian borderline group (31.25\%, 5/16), although this was not statistically significant $(P>0.05)$. The positive expression rate of KRT80 in the ovarian borderline group $(75.00 \%, 12 / 16)$ was higher than that in the normal $(26.67 \%, 4 / 15)$ and benign $(28.57 \%$, $4 / 14)$ groups $(P<0.05)$. The high expression rate of KRT80 in the borderline tumor group $(31.25 \%, 5 / 16)$ was also higher than that in the normal $(0)(P<0.05)$ and benign $(7.14 \%, 1 / 14)(P>0.05)$ groups (Table 1$)$ (Figure 1B). According to the data in the Oncomine database, KRT80 is overexpressed in many different cancer tissues (Figure 1C). In the GEPIA database, the expression of KRT80 in 426 ovarian cancer patients was significantly higher than that in 88 normal ovarian tissue samples $(P<0.05)$ (Figure 1D). In the cBioPortal database, 587 cases of ovarian cancer with KRT80 gene changes were analyzed, and the mutation rate of KRT80 was $4.6 \%$, including $2.39 \%$ amplification, $0.17 \%$ deep deletion, and $2.04 \%$ high mRNA in TCGA (Firehose Legacy) (Figure 1E).

A total of 102 ovarian cancer specimens were evaluated in the present study. The relationship between the expression of KRT80 and clinicopathologic parameters is shown in Table 2. A high expression rate of KRT80 was significantly correlated with FIGO stage and lymph node metastasis $(P<0.01)$. The high expression rate of KRT80 was $69.49 \%$ in the late FIGO group (stage III IV), which was higher than that in the early FIGO group (stage I II) $(39.53 \%)$. The positive rate of KRT80 in the lymph node metastasis group (81.82\%) was higher than that in non-metastasis group $(44.62 \%)$. However, KRT80 expression was not correlated with age, histological grade, or pathological type $(P>0.05)$. 
A
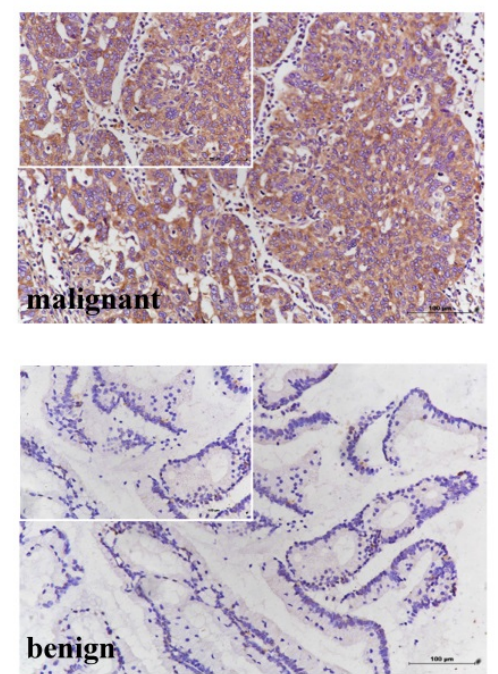

B

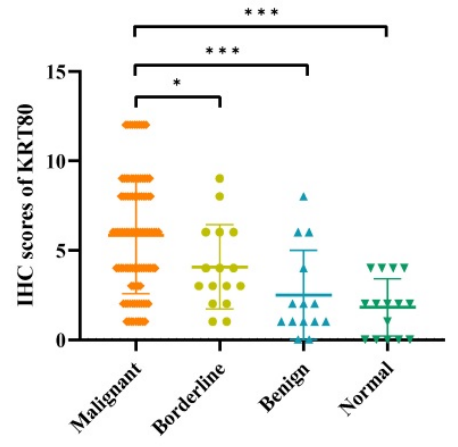

F

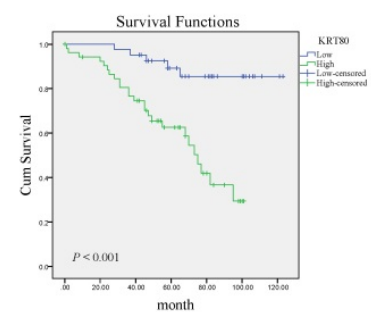

I

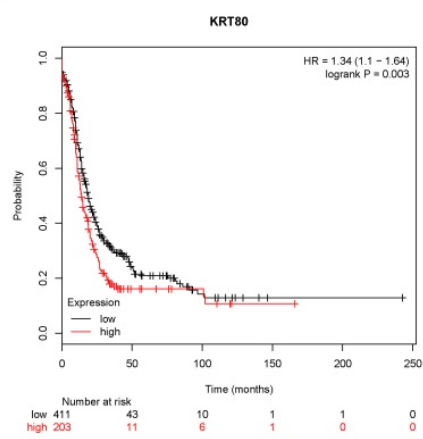

G

J
E
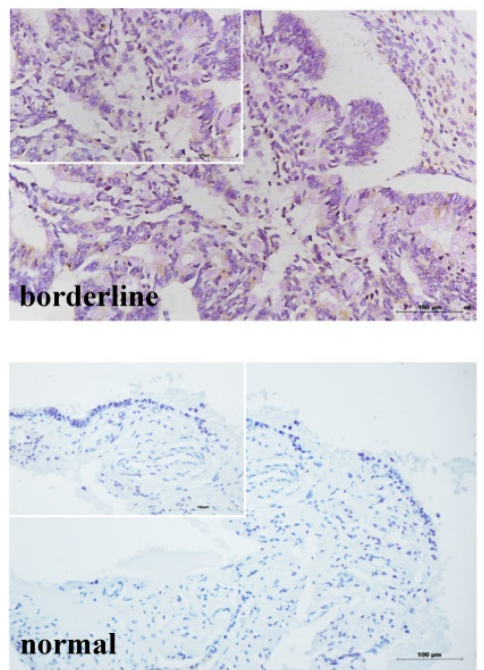

C
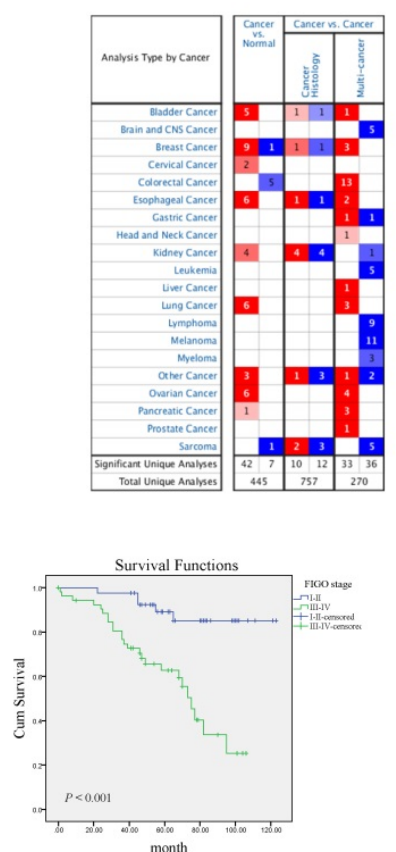

H

D
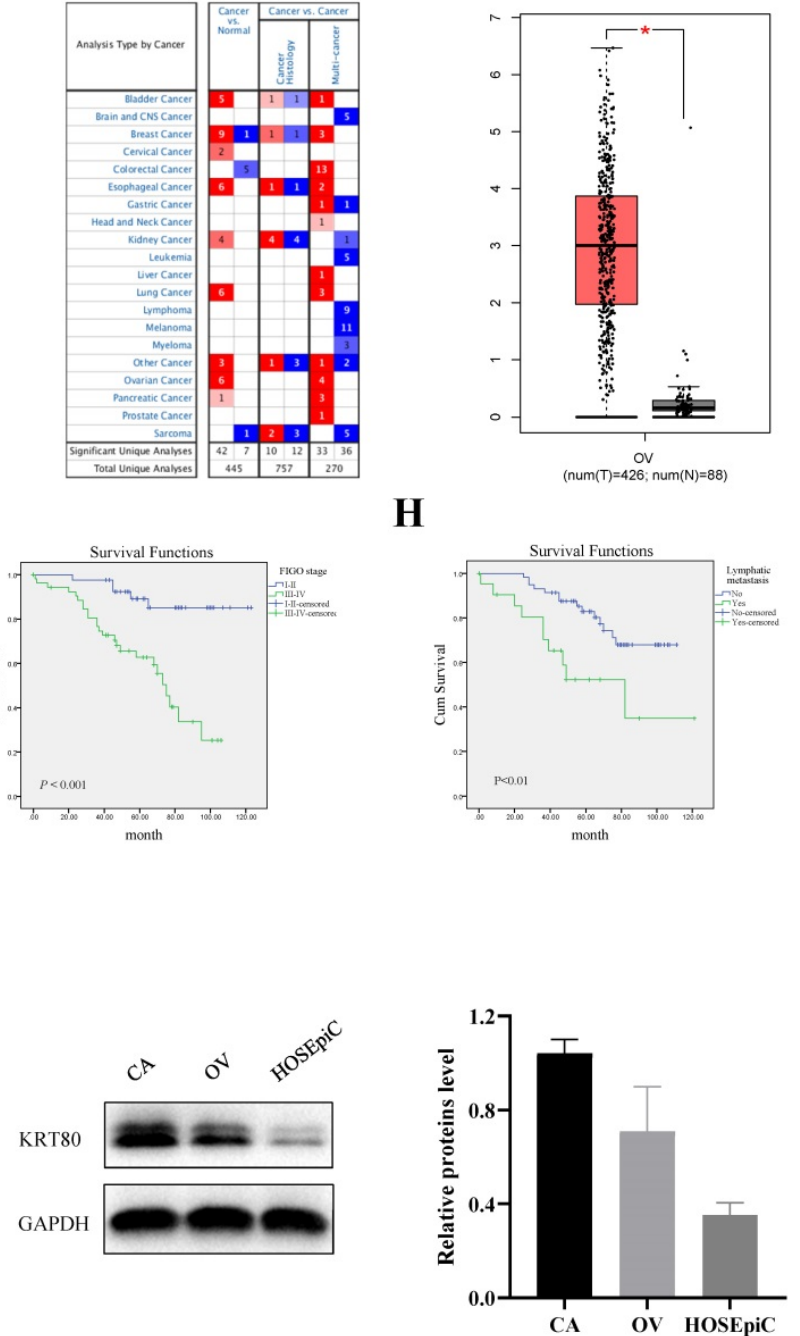

Figure 1. High KRT80 expression in patients with ovarian cancer associated with poor prognosis. (A) KRT80 expression in ovarian tissue samples $(\times 200$, scale bar $=100 \mu \mathrm{m}$; upper left $\times 400)$ : ovarian malignant tumor $(\mathrm{n}=102)$, ovarian borderline tumor $(\mathrm{n}=16)$, ovarian benign tumor $(\mathrm{n}=14)$, ovarian normal tissue $(\mathrm{n}=15)$. $(\mathbf{B})$ Immunostaining scores of KRT80 in malignant, borderline, benign, and normal ovarian tissues. (C) KRT80 mRNA expression in the various tumors from Oncomine database. The cell numbers indicate the number of analyses that meet the thresholds. The color intensity (red or blue) is directly proportional to the significance level of upregulation or downregulation. Table header was divided into Cancer vs. Normal and Cancer vs. Cancer, indicating the differential expression of KRT80 in cancer and normal tissues and in different cancer tissues, respectively. (D) KRT80 mRNA expression in the GEPIA database. Box plots show KRT80 mRNA expression in ovarian tumor (red plot) and the 
corresponding normal tissues (gray plot). Axis unit is $\log _{2}(T P M+1)$. (E) KRT80 genetic variation analysis in cBioPortal (F,G,H). Overall survival analysis according to KRT80 expression, FIGO stage and lymphnode metastasis. (I) KRT80 expression with PFS in Kaplan-Meier Plotter. (J) Representative images and quantitation of the western blotting showed that the protein expression of KRT80 in the ovarian cancer cell lines (CAOV3 and OVCAR3) and normal ovary epithelial cells $(\mathrm{HOSEpiC)}(\mathrm{n}=3)$. GAPDH was used as an internal control. Data are presented as mean \pm SD.
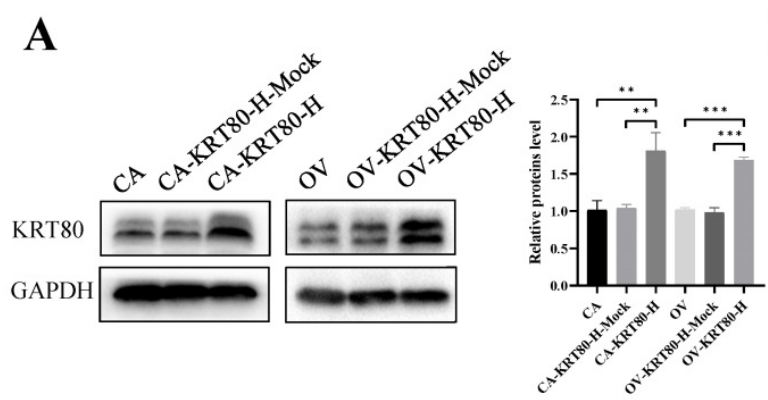

C

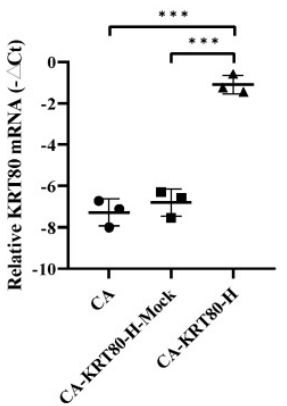

B

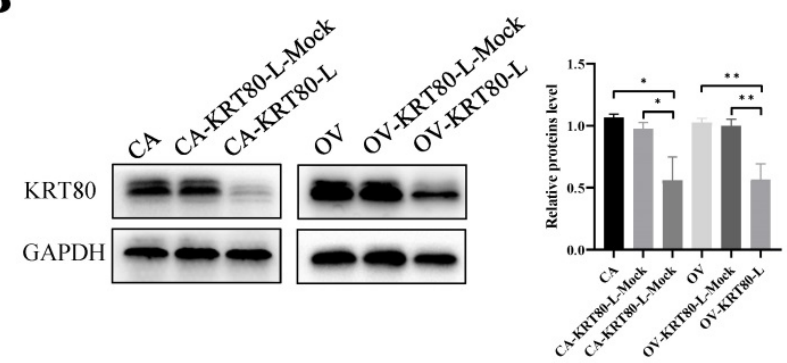

D

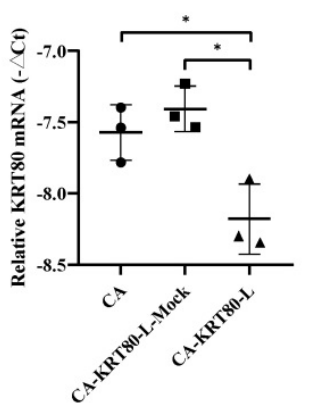

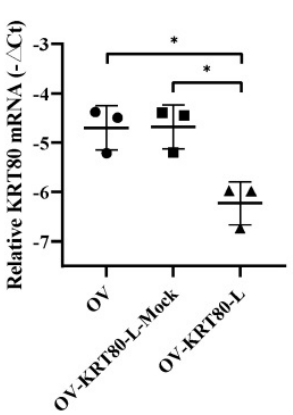

Figure 2. The protein and mRNA expression of KRT80 in the KRT80 overexpression/knockdown groups. (A,B) Representative images and quantitation of the western blotting showed that the protein expression of KRT80 in the KRT80 overexpression/knockdown groups (n=3). GAPDH was used as an internal control. (C,D) The relative KRT80 mRNA expression in the KRT80 overexpression/knockdown groups $(\mathrm{n}=3)$. Data are presented as mean \pm SD. $*, P<0.05 ; * *, P<0.01 ; * * *, P<0.001$.

A total of 102 patients with ovarian cancer were followed up until August 30, 2020. In Kaplan-Meier survival analysis, 102 ovarian cancer patients were divided into low KRT80 expression group (44 cases) and high KRT80 expression group (58 cases); FIGO I-II stage group (43 cases) and FIGO III-IV stage group (59 cases); no lymphatic metastasis group (65 cases) and lymphatic metastasis group (22 cases). The 5 year survival rate of patients with high KRT80 expression was significantly lower than that of patients with low KRT80 expression $(P<0.001)$ (Figure 1F). FIGO stage $(P<0.001)$ (Figure $1 G)$ and lymph node metastasis $(P$ $<0.01$ ) (Figure $1 \mathrm{H}$ ) were significantly correlated with the overall survival, but the age, histological grade, and pathological type were not $(P>0.05)$. In the KM-plot database, patients with high KRT80 expression had significantly shorter progression-free survival than those with low expression of KRT80 $(P<$ 0.01) (Figure 1I).

The relationship between different clinicopathological parameters and prognosis was evaluated by Cox regression analysis. Single-factor Cox regression analysis showed that the expression of KRT80, FIGO stage, and lymph node metastasis were risk factors for the prognosis of ovarian cancer $(P<0.01)$. Multivariate Cox regression analysis showed that KRT80 expression and FIGO stage were independent risk factors for prognosis $(P<0.05)$ (Table 3$)$.

\section{KRT80 promotes ovarian cancer by inducing cell proliferation and cell cycle progression}

The expression of KRT80 protein in ovarian cancer cell lines and normal ovary epithelial cells was detected by western blotting. The results demonstrate that the protein expression level of KRT80 in CAOV3 and OVCAR3 was higher than that in HOSEpiC. Moreover, the expression of KRT80 was highest in CAOV3 cells (Figure 1J).

Overexpression cell lines of KRT80 (CAOV3KRT80-H and OVCAR3-KRT80-H) and knockdown cell lines of KRT80 (CAOV3-KRT80-L and OVCAR3KRT80-L) were constructed using CAOV3 and OVCAR3 cells. Changes in KRT80 expression at the mRNA and protein levels were confirmed using qRTPCR and western blotting, respectively (Figure 2A-D).

Table 1. Expression of KRT80 in different types of ovarian tissue

\begin{tabular}{|c|c|c|c|c|c|c|c|}
\hline \multirow[t]{2}{*}{ Group } & \multirow[t]{2}{*}{ Cases } & \multicolumn{2}{|c|}{ Low expression } & \multicolumn{2}{|c|}{ High expression } & \multirow{2}{*}{$\begin{array}{l}\text { Positive } \\
\text { rate (\%) }\end{array}$} & \multirow{2}{*}{$\begin{array}{l}\text { High expression } \\
\text { rate }(\%)\end{array}$} \\
\hline & & $(-)$ & $(+)$ & $(++)$ & $(+++)$ & & \\
\hline Malignant & 102 & 21 & 23 & 35 & 23 & $79.41^{a, b}$ & $56.86^{c, d}$ \\
\hline Borderline & 16 & 4 & 7 & 4 & 1 & $75.00 \mathrm{e}, \mathrm{f}$ & $31.25 \mathrm{ghh}$ \\
\hline Benign & 14 & 10 & 3 & 1 & 0 & 28.57 & 7.14 \\
\hline Normal & 15 & 11 & 4 & 0 & 0 & 26.67 & 0 \\
\hline \multicolumn{8}{|c|}{$\begin{array}{l}\text { Note: a Malignant vs. benign }\left({ }^{* * *}, P<0.001\right) \text {; b Malignant vs. normal }(* * *, P<0.001) \\
\text { c Malignant vs. benign }(* * *, P<0.001) ; \mathrm{d} \text { Malignant vs. normal }\left({ }^{* * *}, P<0.001\right) ; \mathrm{e} \\
\text { Borderline vs. benign }\left({ }^{*}, P=0.026\right) ; \mathrm{f} \text { Borderline vs. normal }\left({ }^{*}, P=0.012\right) ; \mathrm{g} \\
\text { Borderline vs. benign }(P=0.175) ; \mathrm{h} \text { Borderline vs. normal }\left({ }^{*}, P=0.043\right) .\end{array}$} \\
\hline
\end{tabular}


A

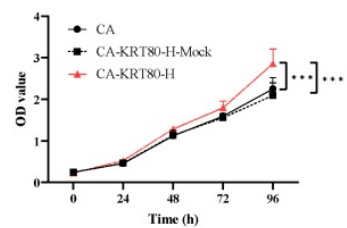

C

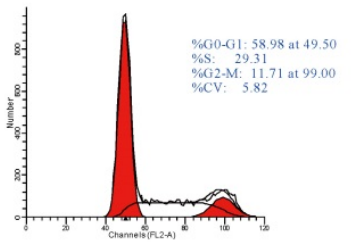

OV

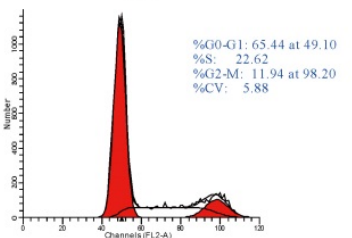

D

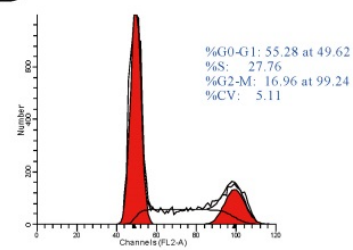

OV

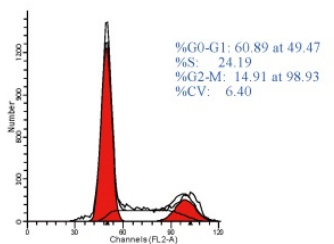

B

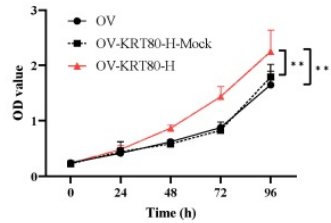

CA-KRT80-H-Mock

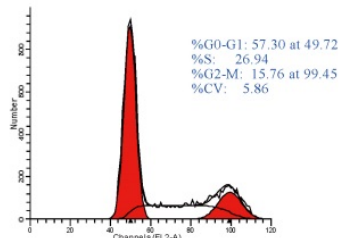

OV-KRT80-H-Mock

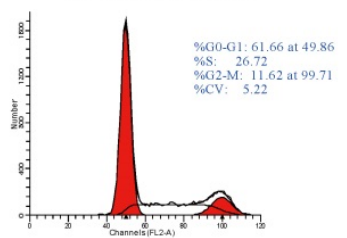

CA-KRT80-L-Mock

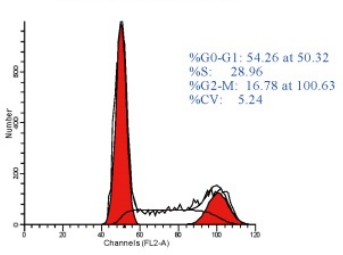

OV-KRT80-L-Mock

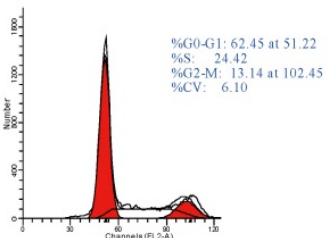

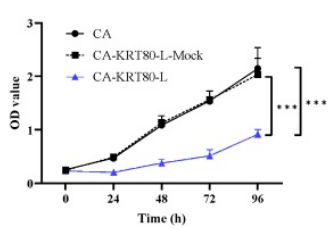

CA-KRT80-H

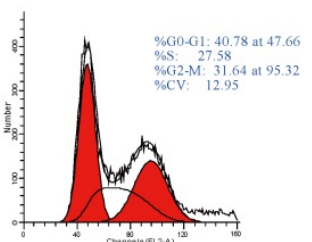

OV-KRT80-H

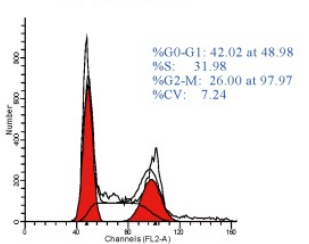

CA-KRT80-L

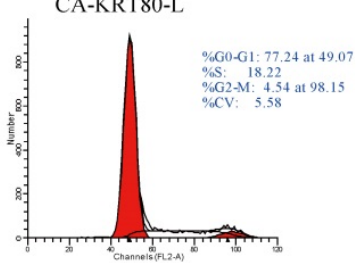

OV-KRT80-L
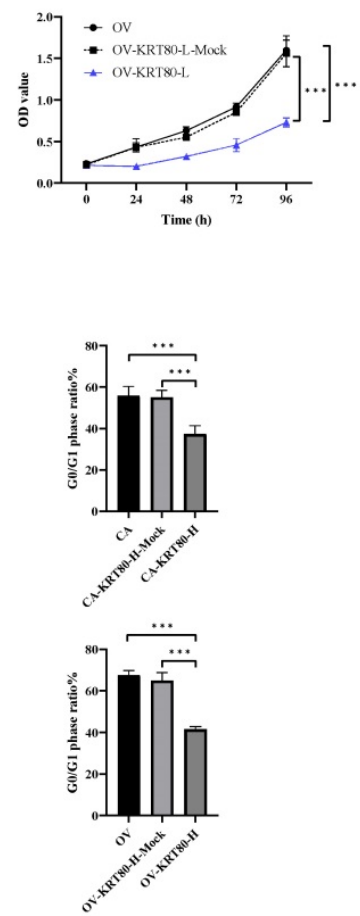
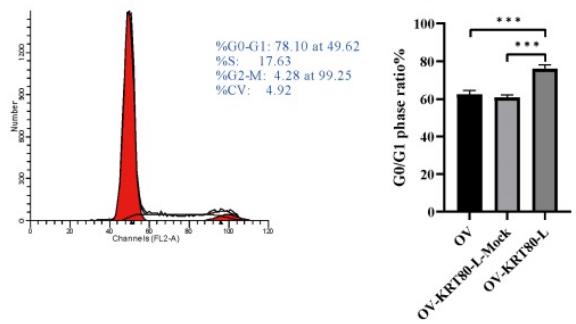

E
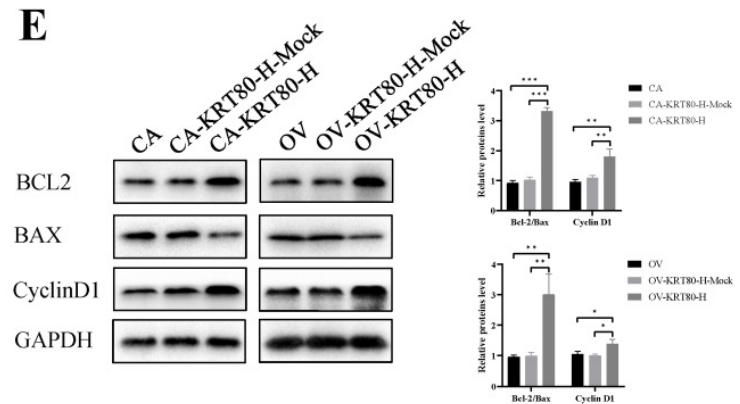

F

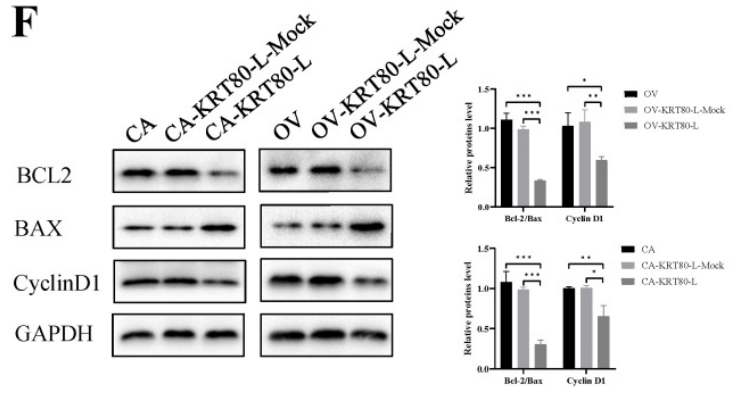

Figure 3. The influences of KRT80 on proliferation and cell cycle in ovarian cancer cells. (A) Overexpression of KRT80 promoted cell proliferation of ovarian cancer cells in CCK8 assay $(n=9)$. (B) Knockdown of KRT80 inhibited cell proliferation of ovarian cancer cells in CCK8 assay $(n=9)$. (C) Overexpression of KRT80 promoted ovarian cancer cell cycle passed into $S$ and $G 2 / M$ phases $(n=6)$. (D) Knockdown of KRT80 duced ovarian cancer cell cycle arrest in the $G 0 / G 1$ phase $(n=6)$. $(E, F)$ Representative images and quantitation of the western blotting showed that the protein expression of BCL2, BAX and CyclinD1 in the KRT80 overexpression/knockdown groups $(n=3)$. GAPDH was used as an internal control. Data are presented as mean \pm SD. *, $P<0.05 ; * *, P<0.01 ; * * *, P<0.001$.

The progression of these cell lines was evaluated by measuring cell proliferation rates and cell cycle arrest after KRT80 overexpression/knockdown. The
CCK8 assay showed that KRT80 overexpression could significantly increase the proliferation rate of cells $(P<$ 0.05 ) (Figure $3 \mathrm{~A}$ ). In contrast, the cell proliferation rate 
after KRT80 knockdown was significantly lower than that in the control group $(P<0.05)$ (Figure 3B). KRT80 overexpression promoted changes in the cell cycle from G1 phase to $S$ phase $(P<0.05)$ (Figure $3 C$ ). Conversely, KRT80 knockdown induced cell cycle arrest in the G0/G1 phase $(P<0.05)$ (Figure 3D). The overexpression of KRT80 increased the expression of BCL2 and CyclinD1, but decreased the expression of BAX (Figure 3E). In contrast, after KRT80 knockdown, the expression of BCL2 and CyclinD1 decreased and the expression of BAX increased (Figure 3F). These results suggest that high KRT80 expression promotes the proliferation of ovarian cancer cells, and the transition of ovarian cancer cells from G1 phase to $S$ phase.

\section{KRT80 promotes invasion and migration of ovarian cancer cells}

The effects of overexpression/knockdown of KRT80 on cell invasion and migration capabilities were evaluated. KRT80 overexpression could significantly enhance the invasive ability of cells $(P<$ 0.05) (Figure 4A), whereas KRT80 knockdown decreased the invasive ability of cells $(P<0.05)$ (Figure 4B). After KRT80 overexpression, the wound healing speed of the cells was faster than that of the control group, and cell migration was enhanced $(P<$ 0.05) (Figure 4C). After KRT80 knockdown, the wound healing speed was slower than that of the control group, and cell migration decreased $(P<0.05)$ (Figure 4D). With KRT80 overexpression, MMP2, MMP9, and N-cadherin expression were upregulated, whereas E-cadherin expression was downregulated $(P<0.05)$ (Figure 4E). The reverse effect was observed with KRT80 knockdown $(P<0.05)$ (Figure 4F). These results further confirmed that KRT80 promotes the invasion, migration, and EMT of ovarian cancer cells.

\section{KRT80 activates the MEK/ERK signaling pathway}

We used the cBioPortal to screen genes that are co-expressed with KRT80, and then used the David database to perform GO enrichment analysis on the KRT80-related genes. A bubble chart containing the top $20 \mathrm{GO}$ terms (BP/CC/MF) and KEGG pathways related to KRT80 was generated in $\mathrm{R}$ using the package ggplot2 (Figure 5A). In order to further explore the molecular mechanism and biological function of KRT80 in ovarian cancer, we analyzed it. GO analysis of biological processes showed that KRT80-related genes were mainly involved in cell-cell adhesion, intermediate filament cytoskeleton organization and angiogenesis. Cellular component analysis indicated that KRT80-related genes were abundant in the extracellular exosome, intermediate filament and focal adhesion. Molecular function analysis indicated that KRT80-related genes were mainly involved in the function of protein binding, structural molecule activity and structural constituent of cytoskeleton. Furthermore, KEGG analysis showed that KRT80-related genes were enriched in MAPK signaling pathway, focal adhesion, ECM-receptor interaction, proteoglycans in cancer and regulation of actin cytoskeleton signaling pathways. Additionally, further pathway enrichment analysis in the GSEA database has been carried out and consistent results have been obtained. Pathway enrichment analysis suggested that KRT80 was related to the MAPK signaling pathway, focal adhesion, actin cytoskeleton regulation, and ECM-receptor interactions (Figure 5B). These signaling pathways are related to core biological carcinogenic processes. Regulation of focal adhesion and reorganization of actin cytoskeleton are crucial determinants of cell migration. Moreover, ERK/MAPK signaling plays an important role during actin and adhesion modulation which is associated with tumor cell invasion [22]. These findings suggest that KRT80 may play an important role in the development of ovarian cancer.

Table 2. Relationship between KRT80 expression and clinicopathological parameters of ovarian epithelial malignant tumors

\begin{tabular}{|c|c|c|c|c|c|c|c|c|c|}
\hline \multirow[t]{2}{*}{ Groups } & \multirow[t]{2}{*}{ Cases } & \multicolumn{2}{|c|}{ Low expression } & \multicolumn{2}{|c|}{ High expression } & \multirow[t]{2}{*}{ Positive rate $(\%)$} & \multirow[t]{2}{*}{$P$} & \multirow[t]{2}{*}{ High expression rate $(\%)$} & \multirow[t]{2}{*}{$P$} \\
\hline & & $\overline{(-)}$ & $(+)$ & $(++)$ & $(+++)$ & & & & \\
\hline \multicolumn{10}{|c|}{ Age at diagnosis (years) } \\
\hline$<53$ & 59 & 15 & 13 & 22 & 9 & 74.58 & \multirow[t]{2}{*}{0.157} & 52.54 & \multirow[t]{2}{*}{0.302} \\
\hline$\geq 53$ & 43 & 6 & 10 & 13 & 14 & 86.05 & & 62.79 & \\
\hline \multicolumn{10}{|l|}{ FIGO stage } \\
\hline I-II & 43 & 11 & 15 & 8 & 9 & 74.42 & \multirow[t]{2}{*}{0.287} & 39.53 & \multirow[t]{2}{*}{$0.003^{* *}$} \\
\hline III-IV & 59 & 10 & 8 & 27 & 14 & 83.05 & & 69.49 & \\
\hline \multicolumn{10}{|l|}{ Differentiation } \\
\hline Well-moderate & 52 & 12 & 15 & 17 & 8 & 76.92 & \multirow[t]{2}{*}{0.526} & 48.08 & \multirow[t]{2}{*}{0.068} \\
\hline Poor & 50 & 9 & 8 & 18 & 15 & 82.00 & & 66.00 & \\
\hline \multicolumn{10}{|c|}{ Lymphatic metastasis } \\
\hline No & 65 & 18 & 18 & 16 & 13 & 72.31 & \multirow[t]{2}{*}{$0.049^{*}$} & 44.62 & \multirow[t]{2}{*}{$0.002^{* *}$} \\
\hline Yes & 22 & 1 & 3 & 13 & 5 & 95.45 & & 81.82 & \\
\hline
\end{tabular}




\begin{tabular}{|c|c|c|c|c|c|c|c|c|c|}
\hline \multirow[t]{2}{*}{ Groups } & \multirow[t]{2}{*}{ Cases } & \multicolumn{2}{|c|}{ Low expression } & \multicolumn{2}{|c|}{ High expression } & \multirow[t]{2}{*}{ Positive rate (\%) } & \multirow[t]{2}{*}{$P$} & \multirow[t]{2}{*}{ High expression rate (\%) } & \multirow[t]{2}{*}{$P$} \\
\hline & & $(-)$ & $(+)$ & $(++)$ & $(+++)$ & & & & \\
\hline Unknowna & 15 & 2 & 2 & 6 & 5 & 86.66 & & 73.33 & \\
\hline \multicolumn{10}{|l|}{ Pathological type } \\
\hline Serous & 67 & 15 & 11 & 26 & 15 & 77.61 & 0.406 & 61.19 & 0.184 \\
\hline Mucinous & 8 & 1 & 3 & 2 & 2 & 87.50 & & 50.00 & \\
\hline Endometrioid & 19 & 5 & 7 & 4 & 3 & 73.68 & & 36.84 & \\
\hline Clear cell carcinoma & 8 & 0 & 2 & 3 & 3 & 100.00 & & 75.00 & \\
\hline
\end{tabular}

Note: a 15 patients without lymphadenectomy.

A

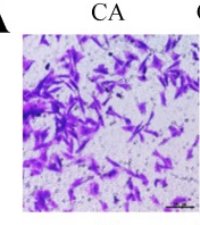

CA

B

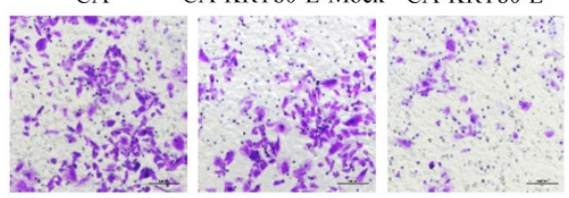

C

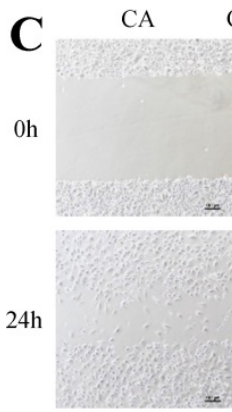

CA-KRT80-H-Mock

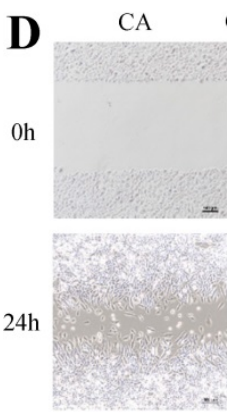

AAT80-L-Mor
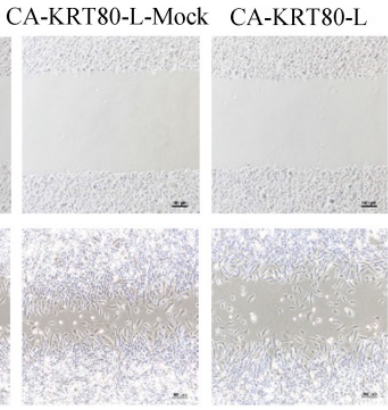

$\mathrm{OV}$

OV-KRT80-H-Mock OV-KRT80-H

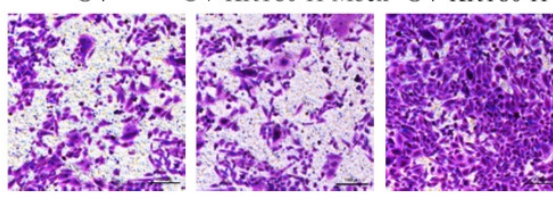

$\mathrm{OV}$

OV-KRT80-L-Mock OV-KRT80-L

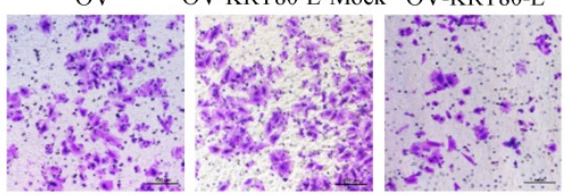

OV

OV-KRT80-H-Mock OV-KRT80-H

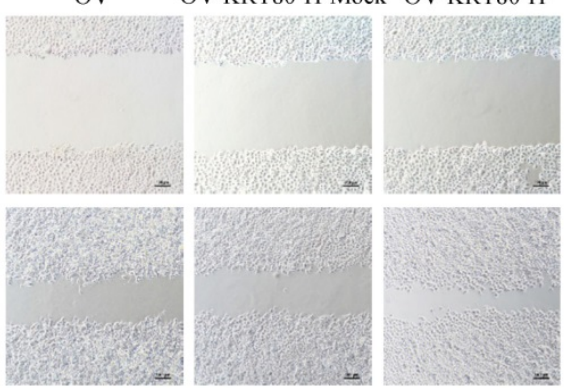

OV

OV-KRT80-L-Mock OV-KRT80-L

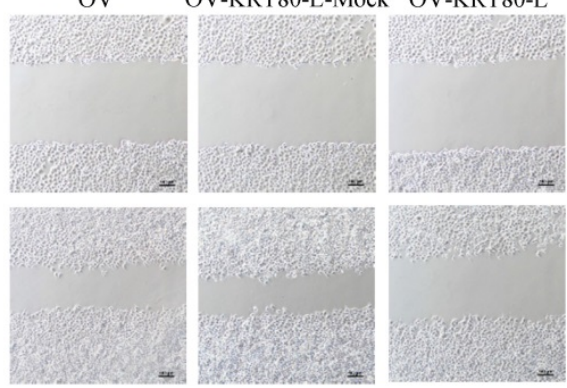

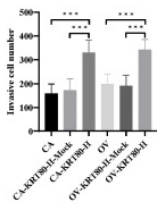
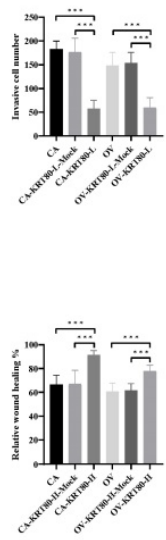

$\mathbf{E}$

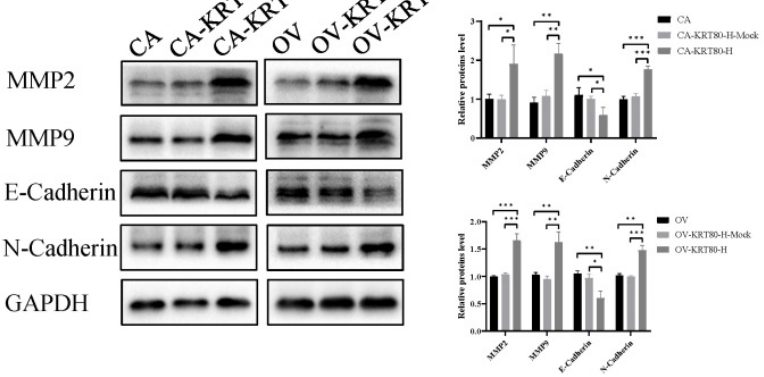

F

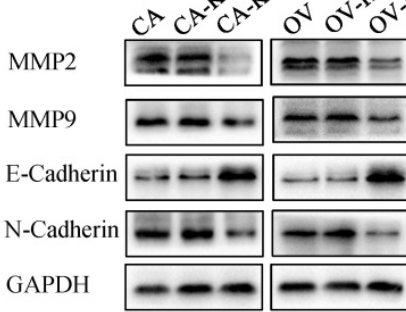

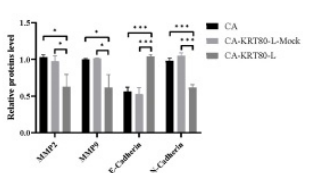

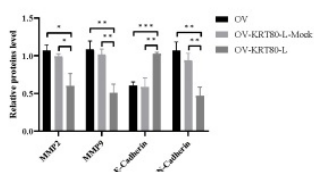

Figure 4. KRT80 promoted invasion and migration in ovarian cancer cells. (A) Overexpression of KRT80 promoted ovarian cancer cells invasion ( $=9 ; \times 200$, scale bar $=100 \mu \mathrm{m})$. (B) Knockdown of KRT80 suppressed ovarian cancer cells invasion $(\mathrm{n}=9 ; \times 200$, scale bar $=100 \mu \mathrm{m})$. (C) Overexpression of KRT80 promoted ovarian cancer cells migration $(n=9 ; \times 100$, scale bar $=100 \mu \mathrm{m})$. (D) Knockdown of KRT80 inhibited ovarian cancer cells migration $(n=9 ; \times 100$, scale bar $=100 \mu \mathrm{m})$. (E,F) Representative images and quantitation of the western blotting showed that the protein expression of MMP2, MMP9, E-cadherin and N-cadherin in the KRT80 overexpression/knockdown groups $(n=3)$. GAPDH was used as an internal control. Data are presented as mean \pm SD. *, $P<0.05 ; * *, P<0.01 ; * * *, P<0.001$. 
A

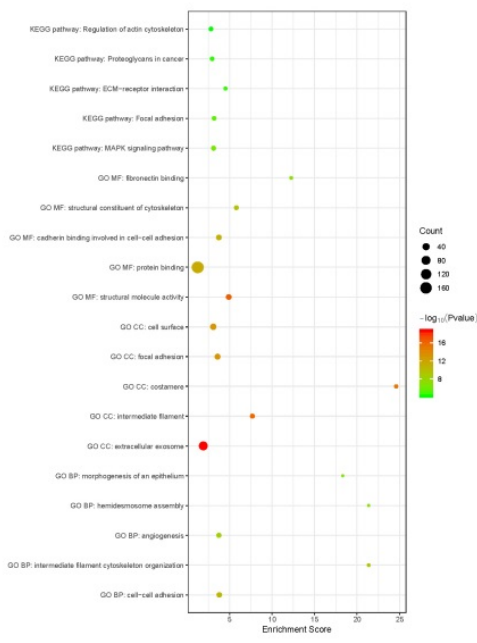

B

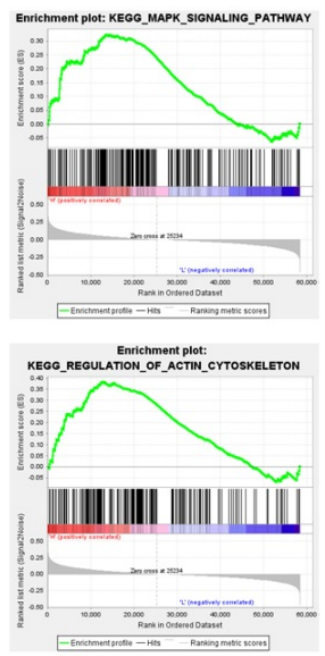

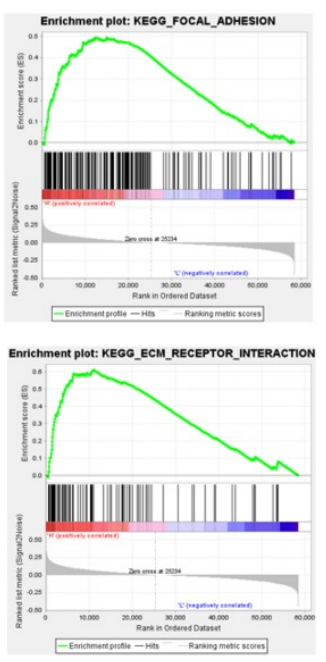

C

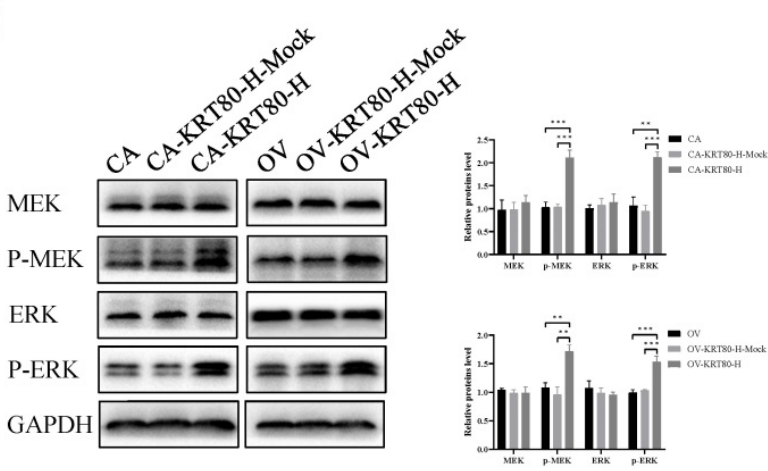

D

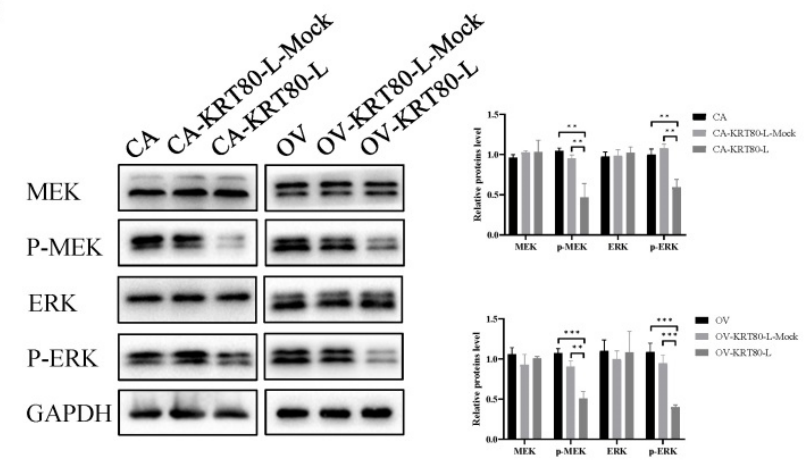

Figure 5. KRT80 activated the MEK/ERK signaling pathway. (A) The bubble plot of top 20 biological functions and pathways of coexpressed genes of KRT80. (B) GSEA analysis of KRT80-related enrichment gene sets. KEGG_MAPK_SIGNALING_PATHWAY (NES $=1.72, P$-value $=0.022$, FDR $=0.083)$; KEGG_FOCAL_ADHESION (NES = $2.28, P$-value $=0.002$, FDR $=0.006)$; KEGG_REGULATION_OF_ACTIN_CYTOSKELETON $(N E S=2.08, P$-value $=0.000$, FDR $=0.022)$; KEGG_ECM_RECEPTOR_ INTERACTION (NES $=2.10, P$-value $=0.002$, FDR $=0.022)$. (C,D) Representative images and quantitation of the western blotting showed that the protein expression of MEK, ERK, $\mathrm{p}$-MEK and $\mathrm{p}$-ERK in the KRT80 overexpression/knockdown groups $(\mathrm{n}=3)$. GAPDH was used as an internal control. Data are presented as mean \pm SD. $*, P<0.05 ; * *, P$ $<0.01 ; * * *, P<0.001$

Table 3. Univariate and Multivariate Cox Analysis of Different Clinicopathological Parameters with Ovarian Cancer

\begin{tabular}{|c|c|c|c|c|c|c|}
\hline \multirow[t]{2}{*}{ Variable } & \multicolumn{3}{|c|}{ Univariate analysis } & \multicolumn{3}{|c|}{ Multivariate analysis } \\
\hline & $\overline{\mathrm{HR}}$ & $\begin{array}{l}95 \% \mathrm{CI} \text { of } \\
\mathrm{HR}\end{array}$ & $P$ & HR & $\begin{array}{l}95 \% \mathrm{CI} \text { of } \\
\mathrm{HR}\end{array}$ & $P$ \\
\hline \multicolumn{7}{|c|}{ Age at diagnosis } \\
\hline & 2.047 & $0.957-4.382$ & 0.065 & & & \\
\hline FIGO stage & & & & & & \\
\hline & 5.904 & $2.237-15.578$ & $<0.001^{* * *}$ & 4.183 & $1.304-13.417$ & $0.016^{*}$ \\
\hline \multicolumn{7}{|l|}{ Differentiation } \\
\hline $\begin{array}{l}\text { Well-moderate } \\
\text { Poor }\end{array}$ & 1.126 & $0.549-2.308$ & 0.746 & & & \\
\hline \multicolumn{7}{|c|}{ Lymphnode metastasis } \\
\hline $\begin{array}{l}\text { No } \\
\text { Yes }\end{array}$ & 3.138 & $1.381-7.133$ & $0.006^{* *}$ & 1.162 & $0.474-2.853$ & 0.743 \\
\hline KRT80 & & & & & & \\
\hline $\begin{array}{l}\text { Low } \\
\text { High }\end{array}$ & 5.627 & $2.145-14.763$ & $<0.001^{* * *}$ & 4.198 & $1.372-12.849$ & $0.012^{*}$ \\
\hline
\end{tabular}

The effect of KRT80 on the MAPK signaling pathway was confirmed via western blotting. KRT80 overexpression increased the expression of p-MEK and p-ERK $(P<0.05)$, but the protein levels of MEK and ERK did not change significantly $(P>0.05)$ (Figure 5C). Similarly, the expression of p-MEK and p-ERK decreased with KRT80 knockdown $(P<0.05)$, but the protein expression levels of MEK and ERK did not change significantly $(P>0.05)$ (Figure 5D). This confirms that KRT80 activates the MEK/ERK signaling pathway.

\section{ETS1 regulates KRT80 expression at the transcriptional level}

We predicted that ETS1 is the transcription factor that regulates KRT80 expression using the Promo and JASPAR databases. ChIP and microarray analysis were used to confirm that ETS1 bound to the promoter $-616 \mathrm{bp}$ to $-486 \mathrm{bp}$ sequence upstream of KRT80 (Figure 6A, B). ETS1 mRNA was positively correlated with KRT80 mRNA in 379 cases of ovarian cancer $(P<0.05, \mathrm{r}=0.161)$ (Figure $6 \mathrm{C})$. These results suggest that ETS1 regulates KRT80 expression at the transcriptional level. 
A

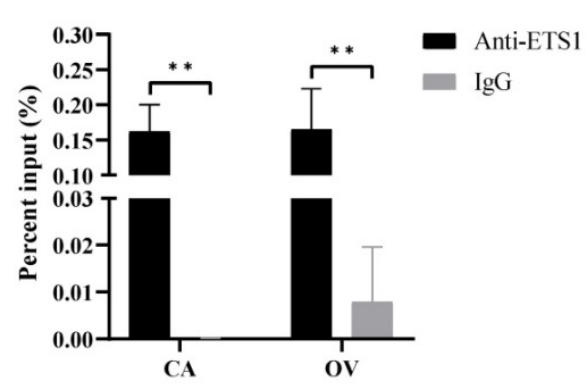

C

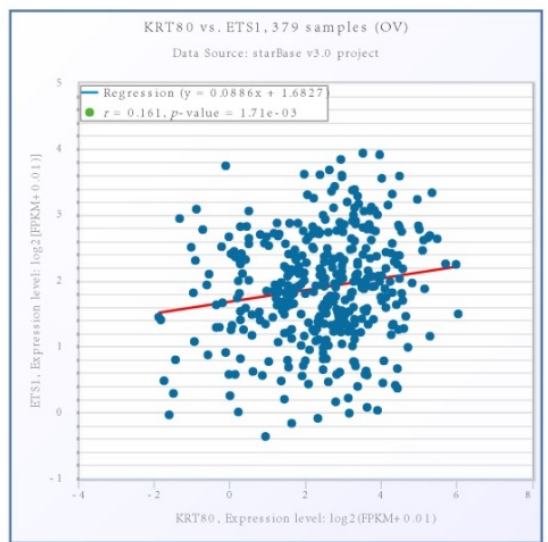

E

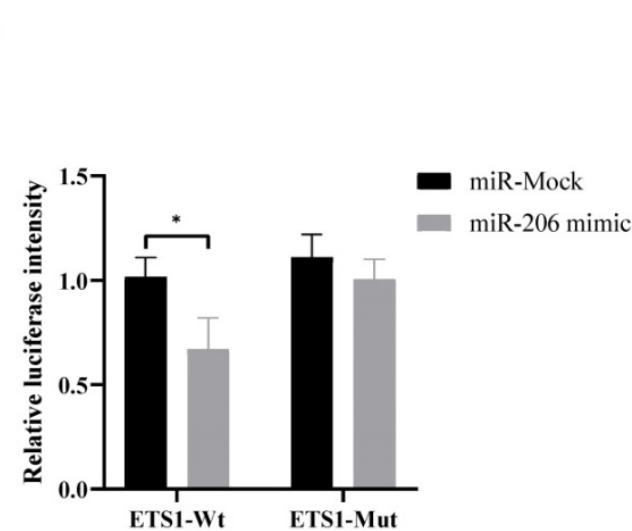

B

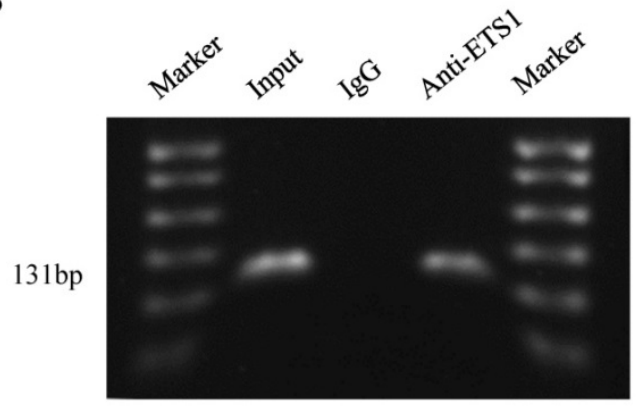

D

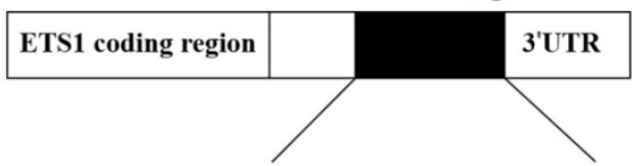

WT ETS1 3'UTR: TGAACTCTTACCTACATTCCA

has-miR-206 : GUGUGUGAAGGAAUGUAAGGU

MUT ETS1 3'UTR: TGAACTCTTACCTTGGGTAAA

F

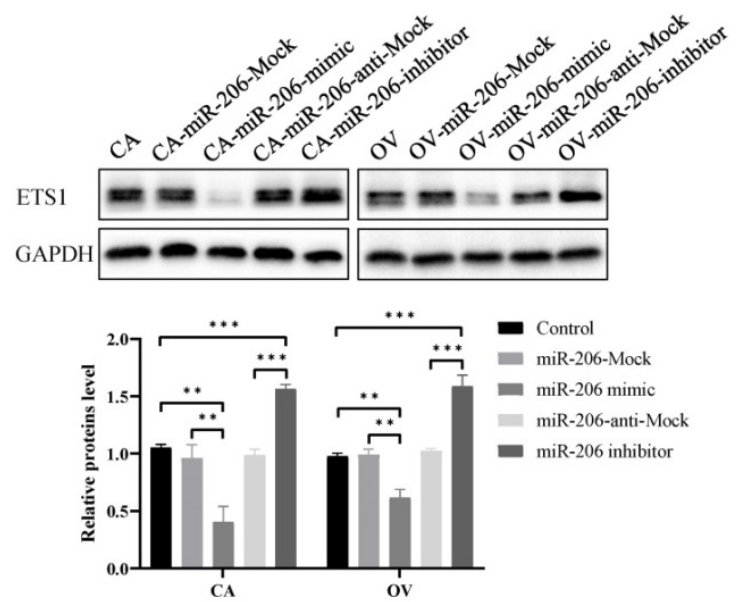

Figure 6. Transcription factor ETSI regulated KRT80 expression and ETS1 is a target of miR-206 in ovarian cancer cell. (A) ChIP-PCR showed that ETS1 could bind the promoter region of KRT80 in ovarian cancer cells using ETS1 primary antibody, IgG was used as a negative control $(n=3)$. (B) ChIP-PCR products were analyzed using horizontal agarose gel electrophoresis and visualized using UV. (C) Correlation between KRT80 mRNA and ETS1 mRNA in 379 ovarian samples using starBase. (D) Binding site between miR-206 and 3'-UTR of ETS1. (E) Dual Luciferase Gene Reporter System for detection of luciferase activity in ETS1-WT or ETS1-MUT and miR-206 mimic or miR-Mock co-transfection. (F) Representative images and quantitation of the western blotting showed that the protein expression of ETS1 in the miR-206 mimics/inhibitor groups $(\mathrm{n}=3)$. GAPDH was used as an internal control. Data are presented as mean \pm SD. *, $P<0.05 ; * *, P<0.01 ; * * *, P<0.001$.

\section{ETS1 is the direct target of miR-206 in ovarian cancer cells}

The TargetScan and miRDB databases together predicted that ETS1 was a potential target for miR-206, with potential binding sites located between the 3'-UTR of ETS1 and miR-206 (Figure 6D). According to a dual luciferase reporter gene assay, the relative luciferase activity of the miR-206 mimic and wild-type ETS1 3'-UTR (ETS1-WT) co-transfected group was significantly lower than that of the control group $(P<0.05)$. However, there was no significant difference in the luciferase activity between the miR-206 mimic and mutant ETS1 3'-UTR (ETS1-MUT) co-transfected group and the control group $(P>0.05)$ (Figure 6E). The protein expression of ETS1 was inhibited after transfection with the miR-206 mimic ( $P$ $<0.05)$. After transfection with the miR-206 inhibitor, ETS1 expression increased $(P<0.05)$ (Figure 6F). The above results confirm that ETS1 is the direct target of 
miR-206 in ovarian cancer cells. In addition, our bioinformatics analysis of multiple databases showed that KRT80 was not a potential target for miR-206. Meanwhile, we detected by western blotting and founded that the protein expression level of KRT80 did not change significantly after transfection with the miR-206 mimic and miR-206 inhibitor $(\mathrm{P}>0.05)$ (Figure S1). Therefore, we speculate that miR-206 has no direct effect on KRT80, but through ETS1 which is a direct target of miR-206 in ovarian cancer cells. And ETS1 further regulates KRT80 expression at the transcriptional level.

\section{Discussion}

Ovarian cancer is the leading cause of death among gynecological malignant tumors. Because of the lack of typical early clinical symptoms and diagnostic methods, it is usually only discovered in the late stages. Intraperitoneal diffusion, extraperitoneal metastasis, and chemotherapy resistance all contribute to the low survival rate of ovarian cancer [3]. Therefore, it is essential to explore the mechanisms by which ovarian cancer develops to enable early screening, prevention, and treatment.

Keratin is an intermediate filament cytoskeletal protein that plays an important role in maintaining the stability and integrity of epithelial cells. Keratin also participates in signal transduction processes, such as intracellular cell stress, proliferation, and metabolism [23, 24]. Keratin is widely present in tumors, plays an important role in the functional regulation of cancer cells, and is a molecular marker of many types of tumors [25-27]. KRT80 exists in almost all types of epithelium, and is a representative marker of epithelial cells. KRT80 expression is related to the advanced differentiation of tissue or cells [7, 9].

To date, there have been few studies on KRT80 expression in malignant tumors. KRT80 is overexpressed in colorectal, gastric, and breast cancers, and esophageal squamous cell carcinoma [11-14, 28], but has not been studied in ovarian cancer. In the present study, we identified the high expression of KRT80 in ovarian cancer tissue by immunohistochemical analysis, and further confirmed this result using cancer databases. The positive expression rate of KRT80 was related to FIGO stage and lymph node metastasis, but not to the age, histological grade, and pathological type of the patient. This could be expanded in other cancer types, given that KRT80 clearly plays a role in ovarian cancer and other cancer types. JL et al. [12] showed that KRT80 expression in colorectal cancer was significantly higher than that in para-cancerous normal tissue, and was significantly correlated with lymph node metastasis and pathological stage. In the present study, Kaplan-Meier analysis showed that KRT80 expression was correlated with the prognosis of ovarian cancer patients, which was consistent with the KM-plot database. KRT80 is an independent risk factor for the prognosis of patients with ovarian cancer. JM et al. [29] found that KRT80 participates in the construction of the miRNA-mRNA network in colorectal cancer exosomes, which is related to the staging, MSI phenotype, and prognosis of colorectal cancer, and plays an important role in the progression of colorectal cancer. Therefore, we concluded that KRT80 plays an important role in the development of ovarian cancer, and may be a prognostic indicator of ovarian cancer.

In the present study, overexpression and knockdown of KRT80 promoted the proliferation of ovarian cancer cells, the change from G0/G1 phase to $S$ phase, and promoted the invasion, migration, and progression of EMT in ovarian cancer cells. These results suggest that KRT80 expression has a cancer-promoting effect in ovarian cancer. JL et al. [12] showed that inhibiting KRT80 expression significantly reduced the survival and proliferation of colorectal cancer cells, suggesting that KRT80 plays an important role in promoting the proliferation of colorectal cancer cells by regulating cell cycle and DNA replication. MW et al. [28] found that inhibition of KRT80 expression in esophageal squamous cell carcinoma inhibited the proliferation, invasion, and migration of esophageal squamous cell carcinoma (ESCC) cells. However, KJ et al. [30] found that the overexpression of TCONS_00049140 in mouse melanocytes downregulated the expression of KRT80, enhanced cell proliferation, and increased melanin production, indicating that KRT80 acts as a tumor suppressor gene in mouse melanoma. Taken together, KRT80 plays an important role in the development of various tumors, and should be studied further in ovarian cancer.

To further explore the mechanisms by which KRT80 affects the malignant biological behavior of ovarian cancer cells, we performed pathway enrichment analysis in the GSEA database and found that KRT80 is related to the MAPK signaling pathway. Mitogen-activated protein kinases (MAPK) are serine/threonine protein kinases that can be activated by different extracellular stimuli. The MAPK pathway plays an important role in cell proliferation, differentiation, apoptosis, angiogenesis, and tumor metastasis [31, 32]. Keratins mediate tumor progression by activating the MEK/ERK signaling pathway. In hepatocellular carcinoma, hepatocyte growth factor activates the c-MET and MEK-ERK1/2 pathways, and upregulates the expression of KRT19. In addition, downstream transcription activators AP1 
and SP1 of ERK1/2 activate the expression of KRT19 in hepatoma cells [33]. Sunitinib reduced the expression of KRT6A by suppressing ERK1/2 and p38 MAPK signal transduction pathways in a skin model [34]. KRT80 promotes tumor progression by activating the AKT signaling pathway. HS et al. [13] found that CIRCPIP5K1A interacts with miR-671-5p to further regulate the expression of KRT80, regulate the proliferation, invasion, and migration of gastric cancer cells, and play a role in carcinogenesis by activating the PI3K/Akt pathway. CL et al. [11] found that KRT80 could interact with DNA-activated catalytic polypeptide (PRKDC) via the AKT pathway to increase the migration and invasion of colorectal cancer cells. In the present study, we verified that the expression of MEK/ERK signaling pathway proteins were dysregulated in ovarian cancer cells with overexpression or knockdown of KRT80. This confirms that KRT80 promotes the malignant biological behavior of ovarian cancer cells by activating the MEK/ERK signaling pathway.

ETS1, a transcription factor with transcriptional activation, is involved in many biological functions, such as tumor cell proliferation, apoptosis, invasion, metastasis, and angiogenesis. At present, many studies have found that ETS1 expression is increased in colorectal, breast, prostate, gastric, and pancreatic cancers, and other malignant tumors [16, 35-38]. A previous study also found that ETS1 is highly expressed in ovarian cancer, and is associated with a poor prognosis [39, 40]. In ovarian cancer, VEGF induces ETS1 expression by activating the PI3K/Akt and p38MAPK signaling pathways, which further activates MMP9 and MMP13 and promotes SKOV3 invasion and metastasis [41]. In addition, transcription factors are involved in the regulation of KRT80 expression during tumor progression. YP et al. [14] found that SREBP1 could target the enhancer of KRT80 and upregulate the expression of KRT80, which could promote cytoskeletal rearrangements at the leading edge, increase focal adhesion and cellular stiffening, and promote the invasion of breast cancer cells. In the present study, we demonstrated for the first time that ETS1 bound at the promoter located $-616 \mathrm{bp}$ to $-486 \mathrm{bp}$ upstream of KRT80 to regulate KRT80 expression. According to the StarBase database, ETS1 mRNA levels are positively correlated with KRT80 mRNA levels. Therefore, we hypothesize that ETS1 regulates KRT80 expression at the transcriptional level.

The miRNA miR-206 is a common tumor suppressor molecule, and its expression is decreased in lung cancer, breast cancer, renal cell carcinoma, endometrial cancer, and many other cancers [42-45]. miRNA can bind to 3'-untranslated regions (3'-UTRs) and affect downstream target expression in cancer [46]. MW et al. [28] found that miR-143-3p affects the malignant phenotype of ESCC cells by targeting KRT80 in esophageal squamous cell carcinoma. The expression level of miR-206 in ovarian cancer is lower than that in normal tissue. KIF2A mRNA contains two binding sites for miR-206, which inhibits the proliferation, migration, and invasion of ovarian cancer cells, and induces apoptosis [47]. In the present study, a dual luciferase reporter assay showed that miR-206 could significantly inhibit the fluorescence intensity of the ETS1-Wt vector, but not the ETS1-Mut vector. This confirms that ETS1 was the direct target of miR-206 in ovarian cancer cells.

In the present study, we confirmed that KRT80 promotes ovarian cancer progression through the MEK/ERK pathway. Interestingly, miR-206 has the potential to promote tumorigenesis through the MEK/ERK pathway. YW et al. [48] found that CircTCF25 induces signal transduction in the MEK/ERK and AKT/mTOR pathways by inhibiting miR-206 expression. HC et al. [49] found that miR-206 activates the MEK/ERK and JNK pathways by regulating IRAK1 to promote LPS-induced inflammatory damage. In addition, previous studies have reported that MER/ERK pathways could regulate ETS1 through its phosphorylation at threonine 38 [50, 51]. Therefore, the effect of KRT80 on the MEK/ERK pathway that we detected may not be independent, but may be a superimposed effect. Meanwhile, we found that miR-206 has no direct effect on KRT80. Therefore, we speculate that miR-206/ETS1 regulates KRT80 to mediate the progression of ovarian cancer through the MEK/ERK pathway.

\section{Conclusion}

In summary, we determined that KRT80 is significantly overexpressed in ovarian cancer and is associated with a poor prognosis. KRT80 overexpression stimulates the MEK/ERK signaling pathway and promotes the proliferation, G0/G1 phase to $S$ phase transition, invasion, and migration of ovarian cancer cells. Mir-206 targets ETS1 to regulate KRT80 expression. We revealed a new cancerpromoting mechanism associated with KRT80. KRT80 is expected to play an important role in the early diagnosis, prognosis evaluation, and clinical treatment of ovarian cancer in the future, and is a promising regulatory gene for ovarian cancer.

\section{Supplementary Material}

Supplementary figure S1.

http://www.jcancer.org/v12p6835s1.pdf 


\section{Acknowledgements}

We acknowledge the contribution of all members who helped with this study.

\section{Funding}

This work was supported by National Natural Science Foundation of China (No. 81672590, No. 81472437) and Outstanding Scientific Fund of Shengjing Hospital (201804).

\section{Ethics approval and consent to participate}

Ethical approval was obtained from the Ethics Review Committee of Shengjing Hospital, China Medical University (the ethical approval code is 2019PS167K). All patients gave informed consent and agreed to participate in the study.

\section{Availability of data and materials}

The datasets used or analyzed during the current study are available from the corresponding author upon reasonable request.

\section{Authors' contributions}

$\mathrm{OL}, \mathrm{CW}$ and $\mathrm{BL}$ conceived and designed the experiments. SW, OL and $\mathrm{YH}$ performed the experiments. RG, HD and SL performed data mining and analysis. OL and XL contributed to writing the manuscript. All authors have read and approved the final manuscript.

\section{Competing Interests}

The authors have declared that no competing interest exists.

\section{References}

1. Jiang W, Chetry M, Pan S, Wang L, Zhu X. Overexpression of Galectin10 Predicts a Better Prognosis in Human Ovarian Cancer. Journal of Cancer. 2021; 12: 2654-64.

2. Siegel RL, Miller KD, Jemal A. Cancer statistics, 2020. CA: a cancer journal for clinicians. 2020; 70: 7-30.

3. Torre LA, Trabert B, DeSantis CE, Miller KD, Samimi G, Runowicz CD, et al. Ovarian cancer statistics, 2018. CA: a cancer journal for clinicians. 2018; 68: 284-96.

4. Yi H, Su YZ, Lin R, Zheng XQ, Pan D, Lin DM, et al. Downregulation of RIPK4 Expression Inhibits Epithelial-Mesenchymal Transition in Ovarian Cancer through IL-6. Journal of immunology research. 2021; 2021: 8875450.

5. Strnad P, Paschke S, Jang KH, Ku NO. Keratins: markers and modulators of liver disease. Current opinion in gastroenterology. 2012; 28: 209-16.

6. Rogers MA, Edler L, Winter H, Langbein L, Beckmann I, Schweizer J. Characterization of new members of the human type II keratin gene family and a general evaluation of the keratin gene domain on chromosome 12q13.13. The Journal of investigative dermatology. 2005; 124: 536-44.

7. Uenishi T, Kubo S, Yamamoto T, Shuto T, Ogawa M, Tanaka H, et al. Cytokeratin 19 expression in hepatocellular carcinoma predicts early postoperative recurrence. Cancer science. 2003; 94: 851-7.

8. Langbein L, Eckhart L, Rogers MA, Praetzel-Wunder S, Schweizer J. Against the rules: human keratin K80: two functional alternative splice variants, K80 and K80.1, with special cellular localization in a wide range of epithelia. The Journal of biological chemistry. 2010; 285: 36909-21.

9. Kurokawa I, Urakawa Y, Senba Y, Kawabata E, Nishimura K, Omoto Y, et al. Keratin profiles may differ between intraepidermal and intradermal invasive eccrine porocarcinoma. Oncology reports. 2006; 16: 473-7.

10. Werner S, Keller L, Pantel K. Epithelial keratins: Biology and implications as diagnostic markers for liquid biopsies. Molecular aspects of medicine. 2020; 72: 100817.
11. Li C, Liu X, Liu Y, Liu X, Wang R, Liao J, et al. Keratin 80 promotes migration and invasion of colorectal carcinoma by interacting with PRKDC via activating the AKT pathway. Cell death \& disease. 2018; 9: 1009

12. Lin J, Fan X, Chen J, Xie X, Yu H. Small interfering RNA-mediated knockdown of KRT80 suppresses colorectal cancer proliferation. Experimental and therapeutic medicine. 2020; 20: 176.

13. Song $\mathrm{H}, \mathrm{Xu} \mathrm{Y}, \mathrm{Xu} \mathrm{T}$, Fan R, Jiang T, Cao M, et al. CircPIP5K1A activates KRT80 and PI3K/AKT pathway to promote gastric cancer development through sponging miR-671-5p. Biomedicine \& pharmacotherapy $=$ Biomedecine \& pharmacotherapie. 2020; 126: 109941.

14. Perone Y, Farrugia AJ, Rodríguez-Meira A, Győrffy B, Ion C, Uggetti A, et al. SREBP1 drives Keratin-80-dependent cytoskeletal changes and invasive behavior in endocrine-resistant ER $\alpha$ breast cancer. Nature communications. 2019; 10: 2115.

15. Wang C, Kam RK, Shi W, Xia Y, Chen X, Cao Y, et al. The Proto-oncogene Transcription Factor Ets1 Regulates Neural Crest Development through Histone Deacetylase 1 to Mediate Output of Bone Morphogenetic Protein Signaling. The Journal of biological chemistry. 2015; 290: 21925-38.

16. Peng C, Gao H, Niu Z, Wang B, Tan Z, Niu W, et al. Integrin av $\beta 6$ and transcriptional factor Ets-1 act as prognostic indicators in colorectal cancer. Cell \& bioscience. 2014; 4: 53.

17. Dittmer J. The role of the transcription factor Ets1 in carcinoma. Seminars in cancer biology. 2015; 35: 20-38.

18. Pan JY, Sun CC, Bi ZY, Chen ZL, Li SJ, Li QQ, et al. miR-206/133b Cluster: A Weapon against Lung Cancer? Molecular therapy Nucleic acids. 2017; 8: 442-9.

19. Liu R, Zheng S, Peng S, Yu Y, Fang J, Tan S, et al. Prognostic Value and Clinicopathological Features of MicroRNA-206 in Various Cancers: A Meta-Analysis. BioMed research international. 2020; 2020: 2159704.

20. Guo Z, Jia H, Ge J. MiR-206 suppresses proliferation and epithelialmesenchymal transition of renal cell carcinoma by inhibiting CDK6 expression. Human cell. 2020; 33: 750-8.

21. Wang Y, Tian Y. miR-206 Inhibits Cell Proliferation, Migration, and Invasion by Targeting BAG3 in Human Cervical Cancer. Oncology research. 2018; 26: 923-31.

22. Carragher NO, Frame MC. Focal adhesion and actin dynamics: a place where kinases and proteases meet to promote invasion. Trends in cell biology. 2004; 14: 241-9.

23. Zhang Q, Shan G, Cao P, He J, Lin Z, Huang Y, et al. Mechanical and biological properties of oxidized horn keratin. Materials science \& engineering $C$, Materials for biological applications. 2015; 47: 123-34.

24. Tang J, Zhuo H, Zhang X, Jiang R, Ji J, Deng L, et al. A novel biomarker Linc00974 interacting with KRT19 promotes proliferation and metastasis in hepatocellular carcinoma. Cell death \& disease. 2014; 5: e1549.

25. Toivola DM, Boor P, Alam C, Strnad P. Keratins in health and disease. Current opinion in cell biology. 2015; 32: 73-81

26. Omary MB, Ku NO, Strnad P, Hanada S. Toward unraveling the complexity of simple epithelial keratins in human disease. The Journal of clinical investigation. 2009; 119: 1794-805.

27. Govaere O, Komuta M, Berkers J, Spee B, Janssen C, de Luca F, et al. Keratin 19: a key role player in the invasion of human hepatocellular carcinomas. Gut. 2014; 63: 674-85.

28. Wada M, Goto $\mathrm{Y}$, Tanaka T, Okada R, Moriya S, Idichi T, et al. RNA sequencing-based microRNA expression signature in esophageal squamous cell carcinoma: oncogenic targets by antitumor miR-143-5p and miR-143-3p regulation. Journal of human genetics. 2020; 65: 1019-34.

29. Ma J, Wang P, Huang L, Qiao J, Li J. Bioinformatic analysis reveals an exosomal miRNA-mRNA network in colorectal cancer. BMC medical genomics. 2021; 14 : 60 .

30. Ji K, Fan R, Zhang J, Yang S, Dong C. Long non-coding RNA expression profile in Cdk5-knockdown mouse skin. Gene. 2018; 672: 195-201.

31. Yuan J, Dong X, Yap J, Hu J. The MAPK and AMPK signalings: interplay and implication in targeted cancer therapy. Journal of hematology \& oncology. 2020; 13: 113.

32. Guo YJ, Pan WW, Liu SB, Shen ZF, Xu Y, Hu LL. ERK/MAPK signalling pathway and tumorigenesis. Experimental and therapeutic medicine. 2020; 19: 1997-2007.

33. Rhee H, Kim HY, Choi JH, Woo HG, Yoo JE, Nahm JH, et al. Keratin 19 Expression in Hepatocellular Carcinoma Is Regulated by Fibroblast-Derived HGF via a MET-ERK1/2-AP1 and SP1 Axis. Cancer research. 2018; 78: 1619-31.

34. Yoshida A, Yamamoto $\mathrm{K}$, Ishida $\mathrm{T}$, Omura $\mathrm{T}$, Itoh $\mathrm{T}$, Nishigori $\mathrm{C}$, et al. Sunitinib decreases the expression of KRT6A and SERPINB1 in 3D human epidermal models. Experimental dermatology. 2021; 30:337-46.

35. Puzovic V, Brcic I, Ranogajec I, Jakic-Razumovic J. Prognostic values of ETS-1, MMP-2 and MMP-9 expression and co-expression in breast cancer patients. Neoplasma. 2014; 61: 439-46.

36. Xu S, Ge J, Zhang Z, Zhou W. MiR-129 inhibits cell proliferation and metastasis by targeting ETS1 via PI3K/AKT/mTOR pathway in prostate cancer. Biomedicine \& pharmacotherapy $=$ Biomedecine $\&$ pharmacotherapie. 2017; 96: 634-41.

37. Nakayama T, Ito M, Ohtsuru A, Naito S, Nakashima M, Fagin JA, et al. Expression of the Ets-1 proto-oncogene in human gastric carcinoma: correlation with tumor invasion. The American journal of pathology. 1996; 149: 1931-9.

38. Cheng K, Feng L, Yu S, Yu C, Chi N. MicroRNA-769-5p Inhibits Pancreatic Ductal Adenocarcinoma Progression by Directly Targeting and 
Downregulating ETS Proto-Oncogene 1. OncoTargets and therapy. 2019; 12: 11737-50.

39. Tomar S, Plotnik JP, Haley J, Scantland J, Dasari S, Sheikh Z, et al. ETS1 induction by the microenvironment promotes ovarian cancer metastasis through focal adhesion kinase. Cancer letters. 2018; 414: 190-204.

40. Bhattacharya R, Ray Chaudhuri S, Roy SS. FGF9-induced ovarian cancer cell invasion involves VEGF-A/VEGFR2 augmentation by virtue of ETS1 upregulation and metabolic reprogramming. J Cell Biochem. 2018; 119: 8174-89.

41. Ghosh S, Basu M, Roy SS. ETS-1 protein regulates vascular endothelial growth factor-induced matrix metalloproteinase- 9 and matrix metalloproteinase-13 expression in human ovarian carcinoma cell line SKOV-3. The Journal of biological chemistry. 2012; 287: 15001-15.

42. Watt K, Newsted D, Voorand E, Gooding RI, Majewski A, Truesdell P, et al. MicroRNA-206 suppresses TGF- $\beta$ signalling to limit tumor growth and metastasis in lung adenocarcinoma. Cellular signalling. 2018; 50: 25-36.

43. Kondo N, Toyama T, Sugiura H, Fujii Y, Yamashita H. miR-206 Expression is down-regulated in estrogen receptor alpha-positive human breast cancer. Cancer research. 2008; 68: 5004-8.

44. Shi J, Zhang D, Zhong Z, Zhang W. IncRNA ROR promotes the progression of renal cell carcinoma through the miR-206/VEGF axis. Molecular medicine reports. 2019; 20: 3782-92.

45. Chen X, Yan Q, Li S, Zhou L, Yang H, Yang Y, et al. Expression of the tumor suppressor miR-206 is associated with cellular proliferative inhibition and impairs invasion in ERa-positive endometrioid adenocarcinoma. Cancer letters. 2012; 314: 41-53.

46. Lagos-Quintana M, Rauhut R, Lendeckel W, Tuschl T. Identification of novel genes coding for small expressed RNAs. Science (New York, NY). 2001; 294: 853-8

47. Sheng $\mathrm{N}, \mathrm{Xu} \mathrm{YZ}, \mathrm{Xi} \mathrm{QH}$, Jiang HY, Wang $\mathrm{CY}$, Zhang $\mathrm{Y}$, et al. Overexpression of KIF2A is Suppressed by miR-206 and Associated with Poor Prognosis in Ovarian Cancer. Cellular physiology and biochemistry : international journal of experimental cellular physiology, biochemistry, and pharmacology. 2018; 50: 810-22.

48. Wang Y, Shi S, Zhang Q, Dong H, Zhang J. MicroRNA-206 upregulation relieves circTCF25-induced osteosarcoma cell proliferation and migration. Journal of cellular physiology. 2020; p:1-10.

49. Chu H, Qu X, Wang F, Chang J, Cheng R, Song X, et al. MicroRNA-206 promotes lipopolysaccharide-induced inflammation injury via regulation of IRAK1 in MRC-5 cells. International immunopharmacology. 2019; 73: 590-8.

50. Taylor MA, Wappett M, Delpuech O, Brown H, Chresta CM. Enhanced MAPK signaling drives ETS1-mediated induction of miR-29b leading to downregulation of TET1 and changes in epigenetic modifications in a subset of lung SCC. Oncogene. 2016; 35: 4345-57.

51. Plotnik JP, Budka JA, Ferris MW, Hollenhorst PC. ETS1 is a genome-wide effector of RAS/ERK signaling in epithelial cells. Nucleic acids research. 2014; 42: 11928-40. 